\title{
A Dual Mixed Formulation for Non-isothermal Oldroyd-Stokes Problem
}

\author{
M. Farhloul ${ }^{1}$ and A. Zine ${ }^{2 *}$ \\ ${ }^{1}$ Département de Mathématiques et de Statistique, Université de Moncton \\ Moncton, N.B., E1A 3E9, Canada \\ ${ }^{2}$ Université de Lyon, Ecole Centrale de Lyon, CNRS UMR 5208 Institut Camille Jordan \\ 36, rue Guy de Collongue, 69134 Ecully, France
}

\begin{abstract}
We propose a mixed formulation for non-isothermal Oldroyd-Stokes problem where the both extra stress and the heat flux's vector are considered. Based on such a formulation, a dual mixed finite element is constructed and analyzed. This finite element method enables us to obtain precise approximations of the dual variable which are, for the non-isothermal fluid flow problems, the viscous and polymeric components of the extra-stress tensor, as well as the heat flux. Furthermore, it has properties analogous to the finite volume methods, namely, the local conservation of the momentum and the mass.
\end{abstract}

Key words: Oldroyd-Stokes problem, non-isotherm, dual mixed formulation AMS subject classification: $65 \mathrm{~N} 30,65 \mathrm{~N} 15,76 \mathrm{D} 07$

\section{Introduction}

We consider a viscoelastic fluid flow in a bounded open domain $\Omega$ in $\mathbb{R}^{2}$ with Lipschitz boundary $\Gamma=\bar{\Gamma}_{D} \cup \bar{\Gamma}_{N}$ such that $\Gamma_{D} \cap \Gamma_{N}=\emptyset$ and meas $\left(\Gamma_{D}\right)>0$. Let $\boldsymbol{u}$ be the velocity vector, $p$ the pressure, $\nabla \boldsymbol{u}$ the gradient velocity tensor, $\boldsymbol{d}(\boldsymbol{u})=(1 / 2)\left(\nabla \boldsymbol{u}+\nabla \boldsymbol{u}^{t}\right)$ the rate of strain tensor, $\boldsymbol{\omega}(\boldsymbol{u})=(1 / 2)\left(\nabla \boldsymbol{u}-\nabla \boldsymbol{u}^{t}\right)$ the vorticity tensor and $\boldsymbol{f}$ the body force.

Assuming the stationary and creeping flow hypotheses, the basic set of momentum and incompressibility equations is given by (see, e.g., [2])

\footnotetext{
*Corresponding author. E-mail: Abdel-Malek.Zine@ec-lyon.fr
} 


$$
\begin{aligned}
-\operatorname{div} \boldsymbol{\sigma}+\nabla p=\boldsymbol{f} & \text { in } \Omega, \\
\operatorname{div} \boldsymbol{u}=0 & \text { in } \Omega .
\end{aligned}
$$

$\boldsymbol{\sigma}$ is the extra-stress tensor which is often splited into a Newtonian part $\boldsymbol{\sigma}_{N}=2 \alpha_{N} \boldsymbol{d}(\boldsymbol{u})$ and a polymeric part $\sigma_{P}$. For the Oldroyd-B model, $\sigma_{P}$ is given by

$$
\boldsymbol{\sigma}_{P}+W_{e}(\boldsymbol{u} \cdot \nabla) \boldsymbol{\sigma}_{P}+W_{e} g_{a}\left(\boldsymbol{\sigma}_{P}, \nabla \boldsymbol{u}\right)-2 \alpha_{P} \boldsymbol{d}(\boldsymbol{u})=0
$$

where $W_{e} \geq 0$ is the Weissenberg number, $0<\alpha_{P}<1$ and $0<\alpha_{N} \leq 1$ are respectively the polymeric and solvent (Newtonian) part of viscosity. $g_{a}(.,$.$) is a bilinear mapping defined by$

$$
g_{a}\left(\boldsymbol{\sigma}_{P}, \nabla \boldsymbol{u}\right)=\boldsymbol{\sigma}_{P} \boldsymbol{\omega}(\boldsymbol{u})-\boldsymbol{\omega}(\boldsymbol{u}) \boldsymbol{\sigma}_{P}-a\left(\boldsymbol{d}(\boldsymbol{u}) \boldsymbol{\sigma}_{P}+\boldsymbol{\sigma}_{P} \boldsymbol{d}(\boldsymbol{u})\right) ;-1 \leq a \leq 1
$$

The various terms appearing in the right hand side of the mapping $g_{a}(\cdot, \cdot)$ are products between matrices.

It is well known that a numerical method for a viscoelastic fluid must be able to handle the case $W_{e}=0$. In that case we obtain the so-called Oldroyd-Stokes's problem

$$
\begin{cases}\boldsymbol{\sigma}_{P}-2 \alpha_{P} \boldsymbol{d}(\boldsymbol{u})=0 & \text { in } \Omega, \\ -\operatorname{div}\left(\boldsymbol{\sigma}_{P}+2 \varepsilon \alpha_{N} \boldsymbol{d}(\boldsymbol{u})\right)+\nabla p=\boldsymbol{f} & \text { in } \Omega, \\ \operatorname{div} \boldsymbol{u}=0 & \text { in } \Omega, \\ \boldsymbol{u}=0 & \text { on } \Gamma\end{cases}
$$

A small real parameter $\varepsilon>0$ is introduced to make the solvent viscosity much smaller than the polymeric part.

Although most of the research on the viscoelastic fluid flows concerns isothermal cases, many flows of practical interest in polymeric melt processing are non-isothermal. The combination of high viscosities of polymeric melts and high deformation rates results in the transformation of large amounts of mechanical energy into heat, and therefore in a temperature rise of the material. This phenomenon is, for instance, used in extruders where viscous dissipation is used to enhance melting of the material [15].

Let $T$ be the temperature, the non-isothermal problem associated with (1.4) is

$$
\begin{cases}\boldsymbol{\sigma}_{P}-2 \alpha_{P}(T) \boldsymbol{d}(\boldsymbol{u})=0 & \text { in } \Omega, \\ -\operatorname{div}\left(\boldsymbol{\sigma}_{P}+2 \varepsilon \alpha_{N}(T) \boldsymbol{d}(\boldsymbol{u})\right)+\nabla p=\boldsymbol{f} & \text { in } \Omega, \\ \operatorname{div} \boldsymbol{u}=0 & \text { in } \Omega, \\ -\operatorname{div}(\kappa \nabla T)+\boldsymbol{u} \cdot \nabla T=Q & \text { in } \Omega, \\ \boldsymbol{u}=0 & \text { on } \Gamma, \\ T=0 & \text { on } \Gamma_{D}, \\ \kappa \nabla T \cdot \boldsymbol{n}=0 & \text { on } \Gamma_{N},\end{cases}
$$

where $\kappa$ is the thermal diffusivity coefficient, and $Q$ the heat source. The dependence of polymer and solvent viscosity upon temperature is given by the following Arrhenius equations: 


$$
\alpha_{P}(T)=a_{1} \exp \left(\frac{b_{1}}{T}\right), \quad \alpha_{N}(T)=a_{2} \exp \left(\frac{b_{2}}{T}\right), \quad b_{1} \in \mathbb{R}^{*} .
$$

The coefficients $a_{1}, b_{1}, a_{2}$ and $b_{2}$ are so that

$$
0<\alpha_{N}(T) \leq 1 \text { and } 0<\alpha_{P}(T)<1 .
$$

We shall also assume the existence of maximum and minimum values for both viscosities,

$$
\alpha_{N, \min } \leq \alpha_{N}(T) \leq \alpha_{N, \max }, \quad \alpha_{P, \min } \leq \alpha_{P}(T) \leq \alpha_{P, \max } .
$$

In the framework of classical finite element approximation, the numerical analysis of problem (1.5) is presented in [6]. The main purpose of this paper is to study a mixed formulation of problem (1.5) where the Newtonian part $\sigma_{N}$ of the extra stress tensor and the heat flux vector are introduced as new unknowns. This means that problem (1.5) is reformulated as

$$
\begin{cases}\boldsymbol{\sigma}_{N}=2 \varepsilon \alpha_{N}(T) \boldsymbol{d}(\boldsymbol{u}) & \text { in } \Omega, \\ \boldsymbol{\sigma}_{P}=2 \alpha_{P}(T) \boldsymbol{d}(\boldsymbol{u}) & \text { in } \Omega, \\ \operatorname{div}\left(\boldsymbol{\sigma}_{N}+\boldsymbol{\sigma}_{P}-p I\right)+\boldsymbol{f}=0 & \text { in } \Omega, \\ \operatorname{div} \boldsymbol{u}=0, & \text { in } \Omega, \\ \boldsymbol{\xi}=\kappa \nabla T-T \boldsymbol{u} & \text { in } \Omega, \\ \operatorname{div} \boldsymbol{\xi}+Q=0 & \text { in } \Omega, \\ \boldsymbol{u}=0 & \text { on } \Gamma, \\ T=0 & \text { on } \Gamma_{D}, \\ \kappa \boldsymbol{\xi} \cdot \boldsymbol{n}=0 & \text { on } \Gamma_{N},\end{cases}
$$

where $I$ is the identity tensor. Based on such a formulation and the PEERS element (see Arnold et al. [1]), we will construct a mixed finite element for problem (1.9). We will analyze this mixed finite element method and prove optimal error estimates.

We close this introduction by pointing out that this work is a first step towards the treatment of non-isothermal viscoelastic fluid flows by a mixed finite element method similar to the one developed here. Naturally, in this case, some upwinding is needed for the convection of the polymeric component of the extra-stress tensor.

An outline of the paper is as follows. In the next section, we present a mixed formulation of problem (1.9). In Section 3, we introduce our finite element approximation and establish the existence of a solution for the discrete problem. The error estimates for all variables are derived in Section 4. In Section 5 we present conclusions.

\section{Mixed formulation}

In order to derive the mixed formulation of problem (1.9), we define the following spaces: 


$$
\begin{aligned}
& \Sigma=\left\{\underset{\sim}{\boldsymbol{\tau}}=\left(\boldsymbol{\tau}_{N}, \boldsymbol{\tau}_{P}, q\right) \in\left[L^{2}(\Omega)\right]^{2 \times 2} \times\left[L^{2}(\Omega)\right]_{s}^{2 \times 2} \times L_{0}^{2}(\Omega) ; \operatorname{div}\left(\boldsymbol{\tau}_{N}+\boldsymbol{\tau}_{P}-q I\right) \in\left[L^{2}(\Omega)\right]^{2}\right\}, \\
& M=\left\{\underset{\sim}{\boldsymbol{v}}=(\boldsymbol{v}, \theta) \in\left[L^{4}(\Omega)\right]^{2} \times L^{2}(\Omega)\right\}, X=\left\{\boldsymbol{\eta} \in H(\operatorname{div} ; \Omega) ; \boldsymbol{\eta} \cdot \boldsymbol{n}_{\mid \Gamma_{N}}=0\right\}, Y=L^{4}(\Omega),
\end{aligned}
$$

where $\left[L^{2}(\Omega)\right]_{s}^{2 \times 2}$ denotes the space of symmetric tensors and

$$
L_{0}^{2}(\Omega)=\left\{q \in L^{2}(\Omega) ; \int_{\Omega} q d x=0\right\}
$$

The spaces $X$ and $Y$ are equipped with the classical norms of $H($ div $; \Omega)$ and $L^{4}(\Omega)$, respectively. $\Sigma$ and $M$ are equipped with the following norms:

$$
\begin{aligned}
& \|\underset{\sim}{\boldsymbol{\tau}}\|_{\Sigma}=\left(\left\|\boldsymbol{\tau}_{N}\right\|^{2}+\left\|\boldsymbol{\tau}_{P}\right\|^{2}+\|q\|^{2}+\left\|\operatorname{div}\left(\boldsymbol{\tau}_{N}+\boldsymbol{\tau}_{P}-q I\right)\right\|^{2}\right)^{1 / 2} \\
& \|\underset{\sim}{\boldsymbol{v}}\|_{M}=\|\boldsymbol{v}\|_{0,4}+\|\theta\|,
\end{aligned}
$$

where $\|\cdot\|_{0,4}$ denotes the norm on $\left[L^{4}(\Omega)\right]^{2}$ and $\|\cdot\|$ denotes the norm on $\left[L^{2}(\Omega)\right]^{d}, d=1,2,4$. When $d=4$, this norm is associated to the inner product on $\left[L^{2}(\Omega)\right]^{2 \times 2}$ :

$$
(\boldsymbol{\sigma}, \boldsymbol{\tau})=\int_{\Omega} \boldsymbol{\sigma}: \boldsymbol{\tau} d x \quad \text { with } \quad \boldsymbol{\sigma}: \boldsymbol{\tau}=\sum_{i, j=1}^{2} \sigma_{i j} \tau_{i j}
$$

We suppose that $\boldsymbol{f} \in\left[L^{2}(\Omega)\right]^{2}$ and $Q \in L^{2}(\Omega)$. We also use the following notations:

- $|\cdot|_{s, p}$ and $\|\cdot\|_{s, p}$ denote, respectively, the usual semi-norm and norm on the Sobolev space $\left(W^{s, p}(\Omega)\right)^{d}, s \in[0, \infty[, p \in[1, \infty[$ and $d=1,2,4$.

- $\operatorname{as}(\boldsymbol{\tau})=\tau_{21}-\tau_{12}$, for any tensor $\boldsymbol{\tau}=\left(\tau_{i j}\right)_{i, j=1,2}$.

Now, from the equalities $\boldsymbol{\sigma}_{N}=2 \varepsilon \alpha_{N}(T) \boldsymbol{d}(\boldsymbol{u}), \boldsymbol{\sigma}_{P}=2 \alpha_{P}(T) \boldsymbol{d}(\boldsymbol{u})$ and div $\boldsymbol{u}=0$, we get, for $\left(\boldsymbol{\tau}_{N}, \boldsymbol{\tau}_{P}, q\right)$ any element of $\Sigma$,

$$
\begin{aligned}
\left(\frac{1}{2 \varepsilon \alpha_{N}(T)} \boldsymbol{\sigma}_{N}, \boldsymbol{\tau}_{N}\right)+\left(\frac{1}{2 \alpha_{P}(T)} \boldsymbol{\sigma}_{P}, \boldsymbol{\tau}_{P}\right) & =\left(\boldsymbol{d}(\boldsymbol{u}), \boldsymbol{\tau}_{N}+\boldsymbol{\tau}_{P}\right) \\
& =\left(\boldsymbol{d}(\boldsymbol{u}), \boldsymbol{\tau}_{N}+\boldsymbol{\tau}_{P}-q I\right) \\
& =\left(\nabla \boldsymbol{u}-\omega \chi, \boldsymbol{\tau}_{N}+\boldsymbol{\tau}_{P}-q I\right) \\
& =-\left(\operatorname{div}\left(\boldsymbol{\tau}_{N}+\boldsymbol{\tau}_{P}-q I\right), \boldsymbol{u}\right)-\left(\operatorname{as}\left(\boldsymbol{\tau}_{N}\right), \omega\right)
\end{aligned}
$$

where $\omega=\frac{1}{2} \operatorname{curl} \boldsymbol{u}=\frac{1}{2}\left(\frac{\partial u_{2}}{\partial x_{1}}-\frac{\partial u_{1}}{\partial x_{2}}\right)$ and $\chi=\left[\begin{array}{rr}0 & -1 \\ 1 & 0\end{array}\right]$. 
Thus, for $\left(\boldsymbol{\tau}_{N}, \boldsymbol{\tau}_{P}, q\right)$ any element of $\Sigma$ and $(\boldsymbol{v}, \theta)$ any element of $M$, we have

$$
\left\{\begin{array}{l}
\left(\operatorname{div}\left(\boldsymbol{\sigma}_{N}+\boldsymbol{\sigma}_{P}-p I\right), \boldsymbol{v}\right)+\left(\operatorname{as}\left(\boldsymbol{\sigma}_{N}\right), \theta\right)+(\boldsymbol{f}, \boldsymbol{v})=0 \\
\left(\frac{1}{2 \varepsilon \alpha_{N}(T)} \boldsymbol{\sigma}_{N}, \boldsymbol{\tau}_{N}\right)+\left(\frac{1}{2 \alpha_{P}(T)} \boldsymbol{\sigma}_{P}, \boldsymbol{\tau}_{P}\right)+\left(\operatorname{div}\left(\boldsymbol{\tau}_{N}+\boldsymbol{\tau}_{P}-q I\right), \boldsymbol{u}\right)+\left(\operatorname{as}\left(\boldsymbol{\tau}_{N}\right), \omega\right)=0 .
\end{array}\right.
$$

On the other hand, from the equalities $\boldsymbol{\xi}=\kappa \nabla T-T \boldsymbol{u}$ and div $\boldsymbol{\xi}+Q=0$, one gets, for any element $\boldsymbol{\eta}$ of $X$ and $\psi$ any element of $Y$,

$$
\left\{\begin{array}{l}
\frac{1}{\kappa}(\boldsymbol{\xi}, \boldsymbol{\eta})+(\operatorname{div} \boldsymbol{\eta}, T)+\frac{1}{\kappa}(T \boldsymbol{u}, \boldsymbol{\eta})=0 \\
(\operatorname{div} \boldsymbol{\xi}, \psi)+(Q, \psi)=0
\end{array}\right.
$$

Thus, the mixed formulation of problem (1.9) reads as follows:

Find $\underset{\sim}{\boldsymbol{\sigma}}=\left(\boldsymbol{\sigma}_{N}, \boldsymbol{\sigma}_{P}, p\right) \in \Sigma, \underset{\sim}{\boldsymbol{u}}=(\boldsymbol{u}, \omega) \in M, \boldsymbol{\xi} \in X$ and $T \in Y$ such that $\forall \underset{\sim}{\boldsymbol{\tau}}=$ $\left(\boldsymbol{\tau}_{N}, \boldsymbol{\tau}_{P}, q\right) \in \Sigma, \forall \underset{\sim}{\boldsymbol{v}}=(\boldsymbol{v}, \theta) \in M, \forall \boldsymbol{\eta} \in X$ and $\forall \psi \in Y$,

$$
\left\{\begin{array}{l}
\left(\frac{1}{2 \varepsilon \alpha_{N}(T)} \boldsymbol{\sigma}_{N}, \boldsymbol{\tau}_{N}\right)+\left(\frac{1}{2 \alpha_{P}(T)} \boldsymbol{\sigma}_{P}, \boldsymbol{\tau}_{P}\right)+\left(\operatorname{div}\left(\boldsymbol{\tau}_{N}+\boldsymbol{\tau}_{P}-q I\right), \boldsymbol{u}\right)+\left(\operatorname{as}\left(\boldsymbol{\tau}_{N}\right), \omega\right)=0 \\
\left(\operatorname{div}\left(\boldsymbol{\sigma}_{N}+\boldsymbol{\sigma}_{P}-p I\right), \boldsymbol{v}\right)+\left(\operatorname{as}\left(\boldsymbol{\sigma}_{N}\right), \theta\right)+(\boldsymbol{f}, \boldsymbol{v})=0 \\
\frac{1}{\kappa}(\boldsymbol{\xi}, \boldsymbol{\eta})+(\operatorname{div} \boldsymbol{\eta}, T)+\frac{1}{\kappa}(T \boldsymbol{u}, \boldsymbol{\eta})=0 \\
(\operatorname{div} \boldsymbol{\xi}, \psi)+(Q, \psi)=0
\end{array}\right.
$$

It is clear that if $\left(\boldsymbol{u}, p, \boldsymbol{\sigma}_{P}, T\right)$ is a solution of problem (1.5) (see [6]), then

$\left(\left(\boldsymbol{\sigma}_{N}, \boldsymbol{\sigma}_{P}, p\right) ;(\boldsymbol{u}, \omega) ; \boldsymbol{\xi} ; T\right)$, with $\boldsymbol{\sigma}_{N}=2 \varepsilon \alpha_{N}(T) \boldsymbol{d}(\boldsymbol{u}), \omega=\frac{1}{2} \operatorname{curl} \boldsymbol{u}$ and $\boldsymbol{\xi}=\kappa \nabla T-T \boldsymbol{u}$, is a solution of problem (2.1).

Remark 1. Concerning the existence of solutions to the continuous problem (1.5), to our knowledge, there is only one paper mentioning this fact (see Damak Besbes and Guillopé, [7]). In that work, the authors obtained the existence and local regular solutions for a general non-isothermal three-dimensional viscoelastic fluid flow problem $(W e \neq 0)$.

Remark 2. From the second equation of (2.1), we have $\left(\operatorname{as}\left(\boldsymbol{\sigma}_{N}\right), \theta\right)=0, \forall \theta \in L^{2}(\Omega)$. This is nothing else than the relaxation of the symmetry of $\sigma_{N}$ by a Lagrange multiplier.

Remark 3. The term $(T \boldsymbol{u}, \boldsymbol{\eta})$ has a meaning since $\boldsymbol{u} \in\left[L^{4}(\Omega)\right]^{2}, T \in L^{4}(\Omega)$ and $\boldsymbol{\eta} \in H(\operatorname{div} ; \Omega) \subset\left[L^{2}(\Omega)\right]^{2}$. 


\section{Analysis of the discrete mixed formulation}

From now on, we suppose that $\Omega$ is a plane domain with polygonal boundary. More precisely, it is assumed that $\Omega$ is a simply connected domain and that its boundary $\Gamma$ is the union of a finite number of linear segments $\bar{\Gamma}_{j}, 1 \leq j \leq n_{e}$ ( $\Gamma_{j}$ is assumed to be an open segment). We further fix a partition of $\left\{1, \ldots, n_{e}\right\}$ into two subsets $I_{D}$ and $I_{N}$. The union of the $\Gamma_{j}$ with $j \in I_{D}$ is denoted by $\Gamma_{D}$ and similarly the union of the $\Gamma_{j}$ with $j \in I_{N}$ is denoted by $\Gamma_{N}$. For $j=1, \ldots, n_{e}$, we denote by $\omega_{j}$ the angle at the vertex $S_{j}$ between $\Gamma_{j+1}$ and $\Gamma_{j}\left(\Gamma_{n_{e}+1} \equiv \Gamma_{1}\right)$. We assume that $\Omega$ is convex and that $\omega_{j} \leq \frac{\pi}{2}$, for all $j=1, \ldots, n_{e}$, such that mixed boundary conditions for $T$ occur near $S_{j}$.

Our aim now is to consider the discretization of problem (2.1). Let $\mathcal{T}_{h}, h>0$, be a regular family of triangulations of $\Omega$ into closed triangles (in the sense of Ciarlet [4]). Let $P_{k}(K)$ be the space of polynomials of degree less than or equal to $k$ on $K \in \mathcal{T}_{h}$. We set

$$
R(K)=R T_{0}(K) \oplus \mathbb{R} \operatorname{curl} b_{K},
$$

where $R T_{0}(K)$ is the Raviart-Thomas element of the lowest degree (see [14], [16], [17]), $b_{K}$ the "bubble function" defined by $b_{K}(x)=\lambda_{1}(x) \lambda_{2}(x) \lambda_{3}(x)$, with $\lambda_{1}, \lambda_{2}$ and $\lambda_{3}$ the barycentric coordinates in $K$, and curl $b_{K}=\left(\frac{\partial b_{K}}{\partial x_{2}},-\frac{\partial b_{K}}{\partial x_{1}}\right)$.

Let us consider the following finite-dimensional spaces:

$$
\begin{aligned}
& \Sigma_{h}=\left\{\left(\boldsymbol{\tau}_{N}, \boldsymbol{\tau}_{P}, q\right) \in \Sigma ;\left.q\right|_{K} \in P_{0}(K),\left.\boldsymbol{\tau}_{P}\right|_{K} \in\left[P_{0}(K)\right]^{2 \times 2},\left.\boldsymbol{\tau}_{N}\right|_{K} \in[R(K)]^{2}, \forall K \in \mathcal{T}_{h}\right\}, \\
& M_{h}=\left\{(\boldsymbol{v}, \theta) \in M ;\left.\boldsymbol{v}\right|_{K} \in\left[P_{0}(K)\right]^{2}, \theta \in C^{0}(\bar{\Omega}),\left.\theta\right|_{K} \in P_{1}(K), \forall K \in \mathcal{T}_{h}\right\}, \\
& X_{h}=\left\{\boldsymbol{\eta} \in H(\operatorname{div}, \Omega) ;\left.\boldsymbol{\eta}\right|_{K} \in R T_{0}(K),\left.\boldsymbol{\eta} \cdot \boldsymbol{n}\right|_{e}=0, \forall e \subset \partial K \cap \Gamma_{N}, \forall K \in \mathcal{T}_{h}\right\}, \\
& Y_{h}=\left\{\psi \in Y ;\left.\psi\right|_{K} \in P_{0}(K), \forall K \in \mathcal{T}_{h}\right\},
\end{aligned}
$$

where $e$ denotes an edge of the triangulation.

The finite element approximation of problem (2.1) is defined as follows:

find $\boldsymbol{\sigma}_{h}=\left(\boldsymbol{\sigma}_{N h}, \boldsymbol{\sigma}_{P h}, p_{h}\right) \in \Sigma_{h},{\underset{\sim}{u}}_{h}=\left(\boldsymbol{u}_{h}, \omega_{h}\right) \in M_{h}, \boldsymbol{\xi}_{h} \in X_{h}$ and $T_{h} \in Y_{h}$ such that $\forall \underset{\sim}{\boldsymbol{\tau}}=\left(\boldsymbol{\tau}_{N}, \boldsymbol{\tau}_{P}, q\right) \in \Sigma_{h}, \forall \underset{\sim}{\boldsymbol{v}}=(\boldsymbol{v}, \theta) \in M_{h}, \forall \boldsymbol{\eta} \in X_{h}$ and $\forall \psi \in Y_{h}$, 


$$
\begin{aligned}
& \left(\frac{1}{2 \varepsilon \alpha_{N}\left(T_{h}\right)} \boldsymbol{\sigma}_{N h}, \boldsymbol{\tau}_{N}\right)+\left(\frac{1}{2 \alpha_{P}\left(T_{h}\right)} \boldsymbol{\sigma}_{P h}, \boldsymbol{\tau}_{P}\right)+\left(\operatorname{div}\left(\boldsymbol{\tau}_{N}+\boldsymbol{\tau}_{P}-q I\right), \boldsymbol{u}_{h}\right)+\left(\operatorname{as}\left(\boldsymbol{\tau}_{N}\right), \omega_{h}\right)=0, \\
& \left(\operatorname{div}\left(\boldsymbol{\sigma}_{N h}+\boldsymbol{\sigma}_{P h}-p_{h} I\right), \boldsymbol{v}\right)+\left(\operatorname{as}\left(\boldsymbol{\sigma}_{N h}\right), \theta\right)+(\boldsymbol{f}, \boldsymbol{v})=0 \\
& \frac{1}{\kappa}\left(\boldsymbol{\xi}_{h}, \boldsymbol{\eta}\right)+\left(\operatorname{div} \boldsymbol{\eta}, T_{h}\right)+\frac{1}{\kappa}\left(T_{h} \boldsymbol{u}_{h}, \boldsymbol{\eta}\right)=0, \\
& \left(\operatorname{div} \boldsymbol{\xi}_{h}, \psi\right)+(Q, \psi)=0 .
\end{aligned}
$$

Note that the difference between the approximations of $\boldsymbol{\sigma}_{P}$ and $\boldsymbol{\sigma}_{N}$ comes from the fact that $\boldsymbol{\sigma}_{P h}$ is a symmetric tensor.

Remark 4. Let us mention that the finite-dimensional spaces $\Sigma_{h}$ and $M_{h}$ are similar to the ones introduced in [1] for the construction of a mixed finite element for the elasticity problem (PEERS element).

In view of a decoupled approach of the previous non-linear problem, we first consider a mixed formulation of the convection-diffusion problem. This is given by the two last equations of (3.1). More precisely, for a given $\boldsymbol{u}_{\sim}=\left(\boldsymbol{u}_{h}, \omega_{h}\right) \in M_{h}$ verifying

$$
\exists C>0 \text { independent of } h:\left\|\boldsymbol{u}_{h}\right\|_{L^{\infty}} \leq C,
$$

we consider the following problem: find $\left(\boldsymbol{\xi}_{h}, T_{h}\right) \in X_{h} \times Y_{h}$ such that

$$
\begin{cases}\frac{1}{\kappa}\left(\boldsymbol{\xi}_{h}, \boldsymbol{\eta}\right)+\left(\operatorname{div} \boldsymbol{\eta}, T_{h}\right)+\frac{1}{\kappa}\left(T_{h} \boldsymbol{u}_{h}, \boldsymbol{\eta}\right)=0 & \forall \boldsymbol{\eta} \in X_{h}, \\ \left(\operatorname{div} \boldsymbol{\xi}_{h}, \psi\right)+(Q, \psi)=0 & \forall \psi \in Y_{h} .\end{cases}
$$

To show the existence and uniqueness of $\left(\boldsymbol{\xi}_{h}, T_{h}\right) \in X_{h} \times Y_{h}$ solution of (3.3), one needs the following lemma:

Lemma 5. Let $\boldsymbol{f} \in\left[L^{2}(\Omega)\right]^{2}, g \in L^{2}(\Omega), \boldsymbol{u} \in\left[L^{\infty}(\Omega)\right]^{2}, \boldsymbol{\xi}_{h} \in X_{h}$ and $z_{h} \in Y_{h}$ such that

$$
\begin{cases}\frac{1}{\kappa}\left(\boldsymbol{\xi}_{h}, \boldsymbol{\eta}\right)+\left(\operatorname{div} \boldsymbol{\eta}, z_{h}\right)+\frac{1}{\kappa}\left(z_{h} \boldsymbol{u}, \boldsymbol{\eta}\right)=(\boldsymbol{f}, \boldsymbol{\eta}) & \forall \boldsymbol{\eta} \in X_{h}, \\ \left(\operatorname{div} \boldsymbol{\xi}_{h}, \psi\right)=(g, \psi) & \forall \psi \in Y_{h} .\end{cases}
$$

Then, for $h$ small enough, there exists $C>0$, independent on $h$ and $\boldsymbol{u}$ such that

$$
\left\|z_{h}\right\| \leq C h\left(\left\|\boldsymbol{\xi}_{h}\right\|+\left\|\operatorname{div} \boldsymbol{\xi}_{h}\right\|\right)+C(\|\boldsymbol{f}\|+\|g\|)
$$


Proof. First, let $\varphi \in L^{2}(\Omega)$. It is well known (see, e.g., [8]) that the boundary value problem

$$
\begin{cases}-\operatorname{div}(\kappa \nabla \theta)-\boldsymbol{u} \cdot \nabla \theta=\varphi & \text { in } \Omega, \\ \theta=0 & \text { on } \Gamma_{D}, \\ \kappa \nabla \theta \cdot \boldsymbol{n}=0 & \text { on } \Gamma_{N}\end{cases}
$$

admits a unique solution. Furthermore, the assumptions on $\Omega$ yield (cf. [13]) $\theta \in H^{2}(\Omega)$ satisfying the stability condition: $\|\theta\|_{2, \Omega} \leq C\|\varphi\|$.

On the other hand, let $\left(\boldsymbol{\xi}_{h}, z_{h}\right) \in X_{h} \times Y_{h}$ satisfying (3.4). We denote by $R T_{0}(\kappa \nabla \theta) \in X_{h}$ the Raviart-Thomas interpolation of $\kappa \nabla \theta$ (see, e.g., [3, section III.3.3]). Then, by the first equation of (3.4), we have

$$
\begin{aligned}
\left(z_{h}, \varphi\right) & =-\left(z_{h}, \operatorname{div}(\kappa \nabla \theta)\right)-\left(z_{h}, \boldsymbol{u} \cdot \nabla \theta\right) \\
& =-\left(z_{h}, \operatorname{div}\left(R T_{0}(\kappa \nabla \theta)\right)-\left(z_{h}, \boldsymbol{u} \cdot \nabla \theta\right)\right. \\
& =\frac{1}{\kappa}\left(\boldsymbol{\xi}_{h}, R T_{0}(\kappa \nabla \theta)\right)+\frac{1}{\kappa}\left(z_{h} \boldsymbol{u}, R T_{0}(\kappa \nabla \theta)\right)-\left(\boldsymbol{f}, R T_{0}(\kappa \nabla \theta)\right)-\left(z_{h}, \boldsymbol{u} \cdot \nabla \theta\right) .
\end{aligned}
$$

Finally,

$$
\begin{aligned}
\left(z_{h}, \varphi\right) & =\frac{1}{\kappa}\left(\boldsymbol{\xi}_{h}, R T_{0}(\kappa \nabla \theta)-\kappa \nabla \theta\right)+\frac{1}{\kappa}\left(z_{h} \boldsymbol{u}, R T_{0}(\kappa \nabla \theta)-\kappa \nabla \theta\right) \\
& +\left(\boldsymbol{\xi}_{h}, \nabla \theta\right)-\left(\boldsymbol{f}, R T_{0}(\kappa \nabla \theta)\right) .
\end{aligned}
$$

Let $\rho_{h}^{0}$ be the $L^{2}$-projection operator on $\Pi_{K \in \mathcal{T}_{h}} P_{0}(K)$. From the second equation of (3.4), we get $\left(\boldsymbol{\xi}_{h}, \nabla \theta\right)=-\left(\operatorname{div} \boldsymbol{\xi}_{h}, \theta\right)=-\left(\operatorname{div} \boldsymbol{\xi}_{h}, \theta-\rho_{h}^{0} \theta\right)-\left(\operatorname{div} \boldsymbol{\xi}_{h}, \rho_{h}^{0} \theta\right)=-\left(\operatorname{div} \boldsymbol{\xi}_{h}, \theta-\rho_{h}^{0} \theta\right)-\left(g, \rho_{h}^{0} \theta\right)$, and then,

$$
\begin{aligned}
\left(z_{h}, \varphi\right) & =\frac{1}{\kappa}\left(\boldsymbol{\xi}_{h}, R T_{0}(\kappa \nabla \theta)-\kappa \nabla \theta\right)+\frac{1}{\kappa}\left(z_{h} \boldsymbol{u}, R T_{0}(\kappa \nabla \theta)-\kappa \nabla \theta\right) \\
& -\left(\operatorname{div} \boldsymbol{\xi}_{h}, \theta-\rho_{h}^{0} \theta\right)-\left(\boldsymbol{f}, R T_{0}(\kappa \nabla \theta)\right)-\left(g, \rho_{h}^{0} \theta\right) .
\end{aligned}
$$

Using the following inequalities:

(i) $\left(\boldsymbol{f}, R T_{0}(\kappa \nabla \theta)\right)=\left(\boldsymbol{f}, R T_{0}(\kappa \nabla \theta)-\kappa \nabla \theta\right)+\kappa(\boldsymbol{f}, \nabla \theta) \leq C h\|\boldsymbol{f}\||\nabla \theta|_{1, \Omega}+\kappa\|\boldsymbol{f}\|\|\nabla \theta\|$

$$
\leq C\|\boldsymbol{f}\|\|\theta\|_{2, \Omega}
$$

(ii) $\left(g, \rho_{h}^{0} \theta\right) \leq\|g\|\left\|\rho_{h}^{0} \theta\right\| \leq\|g\|\|\theta\|$,

we get,

$$
\begin{aligned}
\left(z_{h}, \varphi\right) & \leq C h\left\|\boldsymbol{\xi}_{h}\right\||\nabla \theta|_{1, \Omega}+C h\|\boldsymbol{u}\|_{L^{\infty}}\left\|z_{h}\right\||\nabla \theta|_{1, \Omega} \\
& +C h\left\|\operatorname{div} \boldsymbol{\xi}_{h}\right\||\theta|_{1, \Omega}+C\|\boldsymbol{f}\|\|\theta\|_{2, \Omega}+C\|g\|\|\theta\| \\
& \leq\left[C h\left(\left\|\boldsymbol{\xi}_{h}\right\|+\left\|\operatorname{div} \boldsymbol{\xi}_{h}\right\|\right)+C h\|\boldsymbol{u}\|_{L^{\infty}}\left\|z_{h}\right\|+C(\|\boldsymbol{f}\|+\|g\|)\right]\|\varphi\| .
\end{aligned}
$$


And consequently,

$$
\begin{aligned}
\left\|z_{h}\right\| & \leq C h\|\boldsymbol{u}\|_{L^{\infty}}\left\|z_{h}\right\|+C h\left(\left\|\boldsymbol{\xi}_{h}\right\|+\left\|\operatorname{div} \boldsymbol{\xi}_{h}\right\|\right)+C(\|\boldsymbol{f}\|+\|g\|), \\
\left(1-C h\|\boldsymbol{u}\|_{L^{\infty}}\right)\left\|z_{h}\right\| & \leq C h\left(\left\|\boldsymbol{\xi}_{h}\right\|+\left\|\operatorname{div} \boldsymbol{\xi}_{h}\right\|\right)+C(\|\boldsymbol{f}\|+\|g\|) .
\end{aligned}
$$

Hence, for $h$ small enough, we get the result:

$$
\left\|z_{h}\right\| \leq C h\left(\left\|\boldsymbol{\xi}_{h}\right\|+\left\|\operatorname{div} \boldsymbol{\xi}_{h}\right\|\right)+C(\|\boldsymbol{f}\|+\|g\|) .
$$

Lemma 6. For $h$ small enough, problem (3.3) has a unique solution $\left(\boldsymbol{\xi}_{h}, T_{h}\right) \in X_{h} \times Y_{h}$. Moreover, there exists $C>0$ independent of $h$, such that:

$$
\left\|\boldsymbol{\xi}_{h}\right\|_{X}+\left\|T_{h}\right\| \leq C\|Q\|
$$

Proof. To show the existence and uniqueness of the solution of problem (3.3), it suffices to show that if $\left(\boldsymbol{\xi}_{h}, T_{h}\right) \in X_{h} \times Y_{h}$ is the solution of the homogeneous problem

$$
\begin{cases}\frac{1}{\kappa}\left(\boldsymbol{\xi}_{h}, \boldsymbol{\eta}\right)+\left(\operatorname{div} \boldsymbol{\eta}, T_{h}\right)+\frac{1}{\kappa}\left(T_{h} \boldsymbol{u}_{h}, \boldsymbol{\eta}\right)=0, & \forall \boldsymbol{\eta} \in X_{h}, \\ \left(\operatorname{div} \boldsymbol{\xi}_{h}, \psi\right)=0, & \forall \psi \in Y_{h},\end{cases}
$$

then $\left(\boldsymbol{\xi}_{h}, T_{h}\right)=(0,0)$.

First, from the second equation of (3.5), we get

$$
\operatorname{div} \boldsymbol{\xi}_{h}=0 .
$$

And then, by Lemma 5, for $h$ small enough,

$$
\left\|T_{h}\right\| \leq C h\left(\left\|\boldsymbol{\xi}_{h}\right\|+\left\|\operatorname{div} \boldsymbol{\xi}_{h}\right\|\right)=C h\left\|\boldsymbol{\xi}_{h}\right\| .
$$

Choosing $\boldsymbol{\eta}=\boldsymbol{\xi}_{h}$, in the first equation of (3.5), we get

$$
\frac{1}{\kappa}\left\|\boldsymbol{\xi}_{h}\right\|^{2}+\left(\operatorname{div} \boldsymbol{\xi}_{h}, T_{h}\right)+\frac{1}{\kappa}\left(T_{h} \boldsymbol{u}_{h}, \boldsymbol{\xi}_{h}\right)=0,
$$

which gives

$$
\left\|\boldsymbol{\xi}_{h}\right\|^{2}=-\left(T_{h} \boldsymbol{u}_{h}, \boldsymbol{\xi}_{h}\right) \leq\left\|\boldsymbol{u}_{h}\right\|_{L^{\infty}}\left\|T_{h}\right\|\left\|\boldsymbol{\xi}_{h}\right\|
$$

and then

$$
\left\|\boldsymbol{\xi}_{h}\right\| \leq\left\|T_{h}\right\|\left\|\boldsymbol{u}_{h}\right\|_{L^{\infty}} \leq\left\|\boldsymbol{u}_{h}\right\|_{L^{\infty}} C h\left\|\boldsymbol{\xi}_{h}\right\| .
$$

As, by (3.2), $\left\|\boldsymbol{u}_{h}\right\|_{L^{\infty}} \leq C$ with $C$ a positive constant independent of $h$, for $h$ small enough, one gets $\boldsymbol{\xi}_{h}=0$ and consequently $T_{h}=0$.

Let us now show the stability condition. From the second equation of (3.3), we obtain

$$
\left(\operatorname{div} \boldsymbol{\xi}_{h}, \psi\right)=-(Q, \psi), \quad \forall \psi \in Y_{h} \Longrightarrow\left\|\operatorname{div} \boldsymbol{\xi}_{h}\right\| \leq\|Q\| .
$$


Following lemma 5, we have (for $h$ small enough)

$$
\left\|T_{h}\right\| \leq C h\left(\left\|\boldsymbol{\xi}_{h}\right\|+\left\|\operatorname{div} \boldsymbol{\xi}_{h}\right\|\right)+C\|Q\|
$$

and then

$$
\left\|T_{h}\right\| \leq C\left(h\left\|\boldsymbol{\xi}_{h}\right\|+\|Q\|\right) .
$$

Choosing $\boldsymbol{\eta}=\boldsymbol{\xi}_{h}$ and $\psi=T_{h}$, in (3.3), we get

$$
\frac{1}{\kappa}\left\|\boldsymbol{\xi}_{h}\right\|^{2}+\frac{1}{\kappa}\left(T_{h} \boldsymbol{u}_{h}, \boldsymbol{\xi}_{h}\right)=\left(Q, T_{h}\right) .
$$

And then

$$
\begin{aligned}
\left\|\boldsymbol{\xi}_{h}\right\|^{2} & =\kappa\left(Q, T_{h}\right)-\left(T_{h} \boldsymbol{u}_{h}, \boldsymbol{\xi}_{h}\right) \\
& \leq\left(\kappa\|Q\|+\left\|\boldsymbol{u}_{h}\right\|_{L^{\infty}}\left\|\boldsymbol{\xi}_{h}\right\|\right)\left\|T_{h}\right\| \\
& \leq C\left(\|Q\|+\left\|\boldsymbol{u}_{h}\right\|_{L^{\infty}}\left\|\boldsymbol{\xi}_{h}\right\|\right)\left(h\left\|\boldsymbol{\xi}_{h}\right\|+\|Q\|\right) .
\end{aligned}
$$

It follows, due to Young inequality,

$$
\begin{aligned}
\left(1-C h\left\|\boldsymbol{u}_{h}\right\|_{L^{\infty}}\right)\left\|\boldsymbol{\xi}_{h}\right\|^{2} & \leq C h\|Q\|\left\|\boldsymbol{\xi}_{h}\right\|+C\|Q\|^{2}+C\left\|\boldsymbol{u}_{h}\right\|_{L^{\infty}}\left\|\boldsymbol{\xi}_{h}\right\|\|Q\| \\
& \leq C h\left(\epsilon_{1}\left\|\boldsymbol{\xi}_{h}\right\|^{2}+\frac{1}{4 \epsilon_{1}}\|Q\|^{2}\right) \\
& +C\|Q\|^{2}+C\left\|\boldsymbol{u}_{h}\right\|_{L^{\infty}}\left(\epsilon_{2}\left\|\boldsymbol{\xi}_{h}\right\|^{2}+\frac{1}{4 \epsilon_{2}}\|Q\|^{2}\right)
\end{aligned}
$$

i.e.,

$$
\left(1-C h\left\|\boldsymbol{u}_{h}\right\|_{L^{\infty}}-C h \epsilon_{1}-C \epsilon_{2}\left\|\boldsymbol{u}_{h}\right\|_{L^{\infty}}\right)\left\|\boldsymbol{\xi}_{h}\right\|^{2} \leq C\left(1+\frac{h}{4 \epsilon_{1}}+\frac{\left\|\boldsymbol{u}_{h}\right\|_{L^{\infty}}}{4 \epsilon_{2}}\right)\|Q\|^{2} .
$$

Let $\epsilon_{2}=\frac{1}{2 C\left\|\boldsymbol{u}_{h}\right\|_{L^{\infty}}}$, then

$$
\left(\frac{1}{2}-C h\left(\left\|\boldsymbol{u}_{h}\right\|_{L^{\infty}}+\epsilon_{1}\right)\right)\left\|\boldsymbol{\xi}_{h}\right\|^{2} \leq C\left(1+\frac{h}{4 \epsilon_{1}}+\frac{C\left\|\boldsymbol{u}_{h}\right\|_{L^{\infty}}^{2}}{2}\right)\|Q\|^{2} .
$$

Hence, for $h$ small enough, we get the desired inequalities:

$$
\left\|\boldsymbol{\xi}_{h}\right\| \leq C\|Q\| \text { and }\left\|T_{h}\right\| \leq C\|Q\| \text {. }
$$

The second inequality, comes from $\left\|T_{h}\right\| \leq C\left(h\left\|\boldsymbol{\xi}_{h}\right\|+\|Q\|\right)$. 


\subsection{Existence}

Let us now come back to the discrete formulation of the non-isothermal Oldroyd-Stokes problem. In the next theorem we will show the existence of a solution to the system (3.1).

Theorem 7. There exists a solution $\left(\underset{\sim}{\boldsymbol{\sigma}_{\boldsymbol{h}}}, \underset{\sim}{\boldsymbol{u}_{h}}, \boldsymbol{\xi}_{h}, T_{h}\right) \in \Sigma_{h} \times M_{h} \times X_{h} \times Y_{h}$ of the discrete problem (3.1) satisfying the stability condition

$$
\left\|\boldsymbol{\sigma}_{\boldsymbol{\sim}}\right\|_{\Sigma}+\left\|\underset{\sim}{\boldsymbol{u}_{h}}\right\|+\left\|\boldsymbol{\xi}_{h}\right\|_{X}+\left\|T_{h}\right\| \leq C(\|\boldsymbol{f}\|+\|Q\|) .
$$

The proof of the theorem is made in several steps.

First, let $\left(\boldsymbol{\xi}_{h}, T_{h}\right)$ be the solution to the convection-diffusion problem (3.3). Define the mapping

$$
\begin{aligned}
F: M_{h} \longrightarrow Y_{h} \\
\\
\boldsymbol{u}_{h} \longrightarrow F\left(\boldsymbol{u}_{\sim}\right)=T_{h} .
\end{aligned}
$$

The Problem (3.1) may be reduced to finding $\boldsymbol{\sigma}_{\sim}=\left(\boldsymbol{\sigma}_{N h}, \boldsymbol{\sigma}_{P h}, p_{h}\right) \in \Sigma_{h}, \underset{\sim}{\boldsymbol{u}_{h}}=\left(\boldsymbol{u}_{h}, \omega_{h}\right) \in M_{h}$ such that

$$
\left\{\begin{array}{l}
\left(\frac{1}{2 \varepsilon \alpha_{N}\left(F\left(\boldsymbol{u}_{\sim}\right)\right)} \boldsymbol{\sigma}_{N h}, \boldsymbol{\tau}_{N}\right)+\left(\frac{1}{2 \alpha_{P}\left(F\left(\underline{\sim}_{h}\right)\right)} \boldsymbol{\sigma}_{P h}, \boldsymbol{\tau}_{P}\right)+ \\
\left(\operatorname{div}\left(\boldsymbol{\tau}_{N}+\boldsymbol{\tau}_{P}-q I\right), \boldsymbol{u}_{h}\right)+\left(a s\left(\boldsymbol{\tau}_{N}\right), \omega_{h}\right)=0, \forall \underset{\sim}{\boldsymbol{\tau}}=\left(\boldsymbol{\tau}_{N}, \boldsymbol{\tau}_{P}, q\right) \in \Sigma_{h}, \\
\left(\operatorname{div}\left(\boldsymbol{\sigma}_{N h}+\boldsymbol{\sigma}_{P h}-p_{h} I\right), \boldsymbol{v}\right)+\left(a s\left(\boldsymbol{\sigma}_{N h}\right), \theta\right)+(\boldsymbol{f}, \boldsymbol{v})=0, \forall \underset{\sim}{\boldsymbol{v}}=(\boldsymbol{v}, \theta) \in M_{h} .
\end{array}\right.
$$

Now define the mapping

$$
\mathcal{D}: \Sigma_{h} \times M_{h} \longrightarrow \Sigma_{h} \times M_{h},
$$

implicitly by $\mathcal{D}(\underset{\sim}{\boldsymbol{r}}, \underset{\sim}{\boldsymbol{w}})=\left(\underset{\sim}{\boldsymbol{\sigma}_{h}},{\underset{\sim}{\sim}}_{h}\right)$ if and only if

$$
\left\{\begin{array}{l}
\left(\frac{1}{2 \varepsilon \alpha_{N}(F(\underset{\sim}{\boldsymbol{w}}))} \boldsymbol{\sigma}_{N h}, \boldsymbol{\tau}_{N}\right)+\left(\frac{1}{2 \alpha_{P}(F(\underset{\sim}{\boldsymbol{w}}))} \boldsymbol{\sigma}_{P h}, \boldsymbol{\tau}_{P}\right)+ \\
\left(\operatorname{div}\left(\boldsymbol{\tau}_{N}+\boldsymbol{\tau}_{P}-q I\right), \boldsymbol{u}_{h}\right)+\left(a s\left(\boldsymbol{\tau}_{N}\right), \omega_{h}\right)=0, \forall \underset{\sim}{\boldsymbol{\tau}_{h}}=\left(\boldsymbol{\tau}_{N}, \boldsymbol{\tau}_{P}, q\right) \in \Sigma_{h}, \\
\left(\operatorname{div}\left(\boldsymbol{\sigma}_{N h}+\boldsymbol{\sigma}_{P h}-p_{h} I\right), \boldsymbol{v}\right)+\left(a s\left(\boldsymbol{\sigma}_{N h}\right), \theta\right)+(\boldsymbol{f}, \boldsymbol{v})=0, \forall \underset{\sim}{\boldsymbol{v}}=(\boldsymbol{v}, \theta) \in M_{h} .
\end{array}\right.
$$

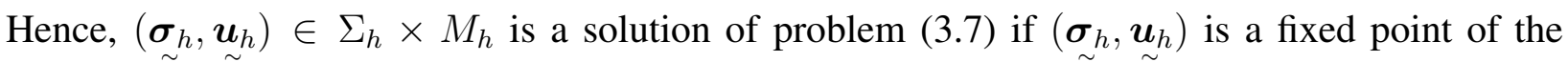
mapping $\mathcal{D}$.

To show the existence of a fixed point of the mapping $\mathcal{D}$, one uses Schaefer's Fixed Point Theorem (see [9], Theorem 4, p. 504). More precisely, the mapping $\mathcal{D}$ must satisfy the following two assumptions:

(H1) $\mathcal{D}$ is continuous and compact,

(H2) the set $\left\{(\underset{\sim}{\boldsymbol{r}}, \underset{\sim}{\boldsymbol{w}}) \in \Sigma_{h} \times M_{h} ;(\underset{\sim}{\boldsymbol{r}}, \underset{\sim}{\boldsymbol{w}})=\lambda \mathcal{D}(\underset{\sim}{\boldsymbol{r}}, \underset{\sim}{\boldsymbol{w}})\right.$ for all $\left.\lambda \in[0,1]\right\}$ is bounded.

We will show $(\mathrm{H} 1)$ and $(\mathrm{H} 2)$ to prove $\mathcal{D}$ has a fixed point. 


\subsubsection{Proof of (H1)}

Let $\left({\underset{\sim}{\boldsymbol{r}}}^{i},{\underset{\sim}{\boldsymbol{w}}}^{i}\right),\left(\boldsymbol{\sigma}_{\sim}^{i},{\underset{\sim}{\boldsymbol{u}}}^{i}\right) \in \Sigma_{h} \times M_{h}, i=1,2$ such that

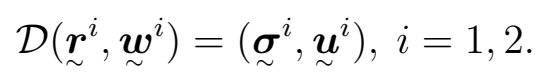

Assume that $\left\|\boldsymbol{w}^{i}\right\|_{L^{\infty}} \leq C, i=1,2$. We will show there exists a constant $C(h)$ such that

$$
\left\|\left({\underset{\sim}{\boldsymbol{\sigma}^{2}}}^{2},{\underset{\sim}{\boldsymbol{u}}}^{2}\right)-\left({\underset{\sim}{\boldsymbol{\sigma}}}^{1},{\underset{\sim}{\boldsymbol{u}}}^{1}\right)\right\|_{\Sigma \times \tilde{M}} \leq C(h)\left\|\left({\underset{\sim}{\boldsymbol{r}}}^{2},{\underset{\sim}{\boldsymbol{w}}}^{2}\right)-\left({\underset{\sim}{\boldsymbol{r}}}^{1},{\underset{\sim}{\boldsymbol{w}}}^{1}\right)\right\|_{\Sigma \times \tilde{M}},
$$

where $\tilde{M}=\left[L^{2}(\Omega)\right]^{2} \times L^{2}(\Omega)$.

To prove (3.9), we begin by estimating $\left\|\boldsymbol{\sim}^{2}-{\underset{\sim}{\boldsymbol{\sigma}}}^{1}\right\|_{\Sigma}$. By definition, $\forall \underset{\sim}{\boldsymbol{\tau}} \in \Sigma_{h}, \forall \underset{\sim}{\boldsymbol{v}} \in M_{h}$ and $i=1,2$, we have

$$
\begin{aligned}
& \left(\frac{1}{2 \varepsilon \alpha_{N}\left(F\left({\underset{\boldsymbol{w}}{i}}^{i}\right)\right)} \boldsymbol{\sigma}_{N}^{i}, \boldsymbol{\tau}_{N}\right)+\left(\frac{1}{2 \alpha_{P}\left(F\left(\underline{\sim}^{i}\right)\right)} \boldsymbol{\sigma}_{P}^{i}, \boldsymbol{\tau}_{P}\right)+\left(\operatorname{div}\left(\boldsymbol{\tau}_{N}+\boldsymbol{\tau}_{P}-q I\right), \boldsymbol{u}^{i}\right)+ \\
& \left(\operatorname{as}\left(\boldsymbol{\tau}_{N}\right), \omega^{i}\right)=0 \\
& \left(\operatorname{div}\left(\boldsymbol{\sigma}_{N}^{i}+\boldsymbol{\sigma}_{P}^{i}-p^{i} I\right), \boldsymbol{v}\right)+\left(\operatorname{as}\left(\boldsymbol{\sigma}_{N}^{i}\right), \theta\right)+(\boldsymbol{f}, \boldsymbol{v})=0 .
\end{aligned}
$$

Then, $\forall \underset{\sim}{\boldsymbol{\tau}} \in \Sigma_{h}$, and $\forall \underset{\sim}{\boldsymbol{v}} \in M_{h}$, we have

$$
\left\{\begin{array}{l}
\left(\frac{1}{2 \varepsilon \alpha_{N}\left(F\left(\underline{\sim}^{2}\right)\right)} \boldsymbol{\sigma}_{N}^{2}-\frac{1}{2 \varepsilon \alpha_{N}\left(F\left(\underline{\sim}^{1}\right)\right)} \boldsymbol{\sigma}_{N}^{1}, \boldsymbol{\tau}_{N}\right)+\left(\frac{1}{2 \alpha_{P}\left(F\left(\boldsymbol{w}^{2}\right)\right)} \boldsymbol{\sigma}_{P}^{2}-\frac{1}{2 \alpha_{P}\left(F\left(\underline{\sim}_{\sim}^{1}\right)\right)} \boldsymbol{\sigma}_{P}^{1}, \boldsymbol{\tau}_{P}\right)+ \\
\left(\operatorname{div}\left(\boldsymbol{\tau}_{N}+\boldsymbol{\tau}_{P}-q I\right), \boldsymbol{u}^{2}-\boldsymbol{u}^{1}\right)+\left(a s\left(\boldsymbol{\tau}_{N}\right), \omega^{2}-\omega^{1}\right)=0 \\
\left(\operatorname{div}\left[\left(\boldsymbol{\sigma}_{N}^{2}+\boldsymbol{\sigma}_{P}^{2}-p^{2} I\right)-\left(\boldsymbol{\sigma}_{N}^{1}+\boldsymbol{\sigma}_{P}^{1}-p^{1} I\right)\right], \boldsymbol{v}\right)+\left(a s\left(\boldsymbol{\sigma}_{N}^{2}-\boldsymbol{\sigma}_{N}^{1}\right), \theta\right)=0 .
\end{array}\right.
$$

The system (3.11) may be written as:

$$
\left\{\begin{array}{l}
\left(\frac{1}{2 \varepsilon \alpha_{N}\left(F\left(\underline{w}^{2}\right)\right)}\left(\boldsymbol{\sigma}_{N}^{2}-\boldsymbol{\sigma}_{N}^{1}\right), \boldsymbol{\tau}_{N}\right)+\left(\frac{1}{2 \alpha_{P}\left(F\left(\underline{\boldsymbol{w}}^{2}\right)\right)}\left(\boldsymbol{\sigma}_{P}^{2}-\boldsymbol{\sigma}_{P}^{1}\right), \boldsymbol{\tau}_{P}\right)+ \\
\left(\operatorname{div}\left(\boldsymbol{\tau}_{N}+\boldsymbol{\tau}_{P}-q I\right), \boldsymbol{u}^{2}-\boldsymbol{u}^{1}\right)+\left(a s\left(\boldsymbol{\tau}_{N}\right), \omega^{2}-\omega^{1}\right)= \\
\left(\left(\frac{1}{2 \varepsilon \alpha_{N}\left(F\left(\underline{\sim}^{1}\right)\right)}-\frac{1}{2 \varepsilon \alpha_{N}\left(F\left(\underline{\sim}^{2}\right)\right)}\right) \boldsymbol{\sigma}_{N}^{1}, \boldsymbol{\tau}_{N}\right)+\left(\left(\frac{1}{2 \alpha_{P}\left(F\left(\underline{\sim}^{1}\right)\right)}-\frac{1}{2 \alpha_{P}\left(F\left(\underline{\sim}^{2}\right)\right)}\right) \boldsymbol{\sigma}_{P}^{1}, \boldsymbol{\tau}_{P}\right), \\
\left(\operatorname{div}\left[\left(\boldsymbol{\sigma}_{N}^{2}+\boldsymbol{\sigma}_{P}^{2}-p^{2} I\right)-\left(\boldsymbol{\sigma}_{N}^{1}+\boldsymbol{\sigma}_{P}^{1}-p^{1} I\right)\right], \boldsymbol{v}\right)+\left(a s\left(\boldsymbol{\sigma}_{N}^{2}-\boldsymbol{\sigma}_{N}^{1}\right), \theta\right)=0 .
\end{array}\right.
$$

Now, choosing $\underset{\sim}{\boldsymbol{\tau}}=\boldsymbol{\sigma}^{2}-{\underset{\sim}{\boldsymbol{\sigma}}}^{1}, \underset{\sim}{\boldsymbol{v}}={\underset{\sim}{\boldsymbol{u}}}^{2}-{\underset{\sim}{\boldsymbol{u}}}^{1}$ in (3.12), and using (1.8), we get the following 
estimates:

$$
\left\{\begin{array}{l}
\frac{1}{2 \varepsilon \alpha_{N, \max }}\left\|\boldsymbol{\sigma}_{N}^{2}-\boldsymbol{\sigma}_{N}^{1}\right\|^{2}+\frac{1}{2 \alpha_{P, \max }}\left\|\boldsymbol{\sigma}_{P}^{2}-\boldsymbol{\sigma}_{P}^{1}\right\|^{2} \\
\leq \frac{1}{2 \varepsilon}\left\|\frac{1}{\alpha_{N}\left(F\left(\boldsymbol{w}^{1}\right)\right)}-\frac{1}{\alpha_{N}\left(F\left(\boldsymbol{w}^{2}\right)\right)}\right\|\left\|\boldsymbol{\sigma}_{N}^{1}\right\|_{L^{\infty}}\left\|\boldsymbol{\sigma}_{N}^{2}-\boldsymbol{\sigma}_{N}^{1}\right\| \\
+\frac{1}{2}\left\|\frac{1}{\alpha_{P}\left(F\left(\boldsymbol{w}^{1}\right)\right)}-\frac{1}{\alpha_{P}\left(F\left(\underline{\sim}^{2}\right)\right)}\right\|\left\|\boldsymbol{\sigma}_{P}^{1}\right\|_{L^{\infty}}\left\|\boldsymbol{\sigma}_{P}^{2}-\boldsymbol{\sigma}_{P}^{1}\right\| .
\end{array}\right.
$$

$\frac{1}{\alpha_{N}(\cdot)}$ and $\frac{1}{\alpha_{P}(\cdot)}$ are Lipschitz continuous functions as they are absolutely bounded exponential functions, then

$$
\begin{aligned}
& \left\|\frac{1}{\alpha_{N}\left(T^{1}\right)}-\frac{1}{\alpha_{N}\left(T^{2}\right)}\right\| \leq C\left\|T^{1}-T^{2}\right\|, \\
& \left\|\frac{1}{\alpha_{P}\left(T^{1}\right)}-\frac{1}{\alpha_{P}\left(T^{2}\right)}\right\| \leq C\left\|T^{1}-T^{2}\right\| .
\end{aligned}
$$

On the other hand, we have the inverse inequalities, obtained if the family of triangulations is supposed to be uniformly regular:

$$
\begin{gathered}
\left\|\boldsymbol{\sigma}_{N}^{1}\right\|_{L^{\infty}} \leq C h^{-1}\left\|\boldsymbol{\sigma}_{N}^{1}\right\|, \\
\left\|\boldsymbol{\sigma}_{P}^{1}\right\|_{L^{\infty}} \leq C h^{-1}\left\|\boldsymbol{\sigma}_{P}^{1}\right\| .
\end{gathered}
$$

Using these inequalities and (3.13), we obtain

$$
\left\{\begin{array}{l}
\frac{1}{2 \varepsilon \alpha_{N, \max }}\left\|\boldsymbol{\sigma}_{N}^{2}-\boldsymbol{\sigma}_{N}^{1}\right\|^{2}+\frac{1}{2 \alpha_{P, \max }}\left\|\boldsymbol{\sigma}_{P}^{2}-\boldsymbol{\sigma}_{P}^{1}\right\|^{2} \\
\leq C h^{-1}\left\|F\left({\underset{\sim}{\sim}}^{2}\right)-F\left({\underset{\sim}{\boldsymbol{w}}}^{1}\right)\right\|\left(\left\|\boldsymbol{\sigma}_{N}^{1}\right\|\left\|\boldsymbol{\sigma}_{N}^{2}-\boldsymbol{\sigma}_{N}^{1}\right\|+\left\|\boldsymbol{\sigma}_{P}^{1}\right\|\left\|\boldsymbol{\sigma}_{P}^{2}-\boldsymbol{\sigma}_{P}^{1}\right\|\right) .
\end{array}\right.
$$

Now, let $\left(\tilde{\boldsymbol{\xi}}^{i}, \tilde{T}^{i}\right) \in X_{h} \times Y_{h}, i=1,2$, be the solution of

$$
\begin{cases}\frac{1}{\kappa}\left(\tilde{\boldsymbol{\xi}}^{i}, \boldsymbol{\eta}\right)+\left(\operatorname{div} \boldsymbol{\eta}, \tilde{T}^{i}\right)+\frac{1}{\kappa}\left(\tilde{T}^{i} \boldsymbol{w}^{i}, \boldsymbol{\eta}\right)=0 & \forall \boldsymbol{\eta} \in X_{h}, \\ \left(\operatorname{div} \tilde{\boldsymbol{\xi}}^{i}, \psi\right)+(Q, \psi)=0 & \forall \psi \in Y_{h} .\end{cases}
$$

Let us recall that $F\left({\underset{\sim}{\boldsymbol{w}}}^{i}\right)=\tilde{T}^{i}, i=1,2$. From (3.15), we have

$$
\begin{cases}\frac{1}{\kappa}\left(\tilde{\boldsymbol{\xi}}^{2}-\tilde{\boldsymbol{\xi}}^{1}, \boldsymbol{\eta}\right)+\left(\operatorname{div} \boldsymbol{\eta}, \tilde{T}^{2}-\tilde{T}^{1}\right)+\frac{1}{\kappa}\left(\tilde{T}^{2} \boldsymbol{w}^{2}-\tilde{T}^{1} \boldsymbol{w}^{1}, \boldsymbol{\eta}\right)=0 & \forall \boldsymbol{\eta} \in X_{h}, \\ \left(\operatorname{div}\left(\tilde{\boldsymbol{\xi}}^{2}-\tilde{\boldsymbol{\xi}}^{1}, \psi\right)=0\right. & \forall \psi \in Y_{h} .\end{cases}
$$

The second equation of (3.16) leads to

$$
\operatorname{div}\left(\tilde{\boldsymbol{\xi}}^{2}-\tilde{\boldsymbol{\xi}}^{1}\right)=0 .
$$


The first equation of (3.16) writes, $\forall \boldsymbol{\eta} \in X_{h}$,

$$
\frac{1}{\kappa}\left(\tilde{\boldsymbol{\xi}}^{2}-\tilde{\boldsymbol{\xi}}^{1}, \boldsymbol{\eta}\right)+\left(\operatorname{div} \boldsymbol{\eta}, \tilde{T}^{2}-\tilde{T}^{1}\right)+\frac{1}{\kappa}\left(\left(\tilde{T}^{2}-\tilde{T}^{1}\right) \boldsymbol{w}^{1}, \boldsymbol{\eta}\right)=\frac{1}{\kappa}\left(\tilde{T}^{2}\left(\boldsymbol{w}^{1}-\boldsymbol{w}^{2}\right), \boldsymbol{\eta}\right) .
$$

Using Lemma 5, we get the following estimation

$$
\left\|\tilde{T}^{2}-\tilde{T}^{1}\right\| \leq C h\left\|\tilde{\boldsymbol{\xi}}^{2}-\tilde{\boldsymbol{\xi}}^{1}\right\|+C\left\|\tilde{T}^{2}\left(\boldsymbol{w}^{1}-\boldsymbol{w}^{2}\right)\right\| .
$$

Choosing, in (3.17), $\boldsymbol{\eta}=\tilde{\boldsymbol{\xi}}^{2}-\tilde{\boldsymbol{\xi}}^{1}$, and using the fact that $\operatorname{div}\left(\tilde{\boldsymbol{\xi}}^{2}-\tilde{\boldsymbol{\xi}}^{1}\right)=0$, we have

$$
\left\|\tilde{\boldsymbol{\xi}}^{2}-\tilde{\boldsymbol{\xi}}^{1}\right\|^{2}+\left(\left(\tilde{T}^{2}-\tilde{T}^{1}\right) \boldsymbol{w}^{1}, \tilde{\boldsymbol{\xi}}^{2}-\tilde{\boldsymbol{\xi}}^{1}\right)=\left(\tilde{T}^{2}\left(\boldsymbol{w}^{1}-\boldsymbol{w}^{2}\right), \tilde{\boldsymbol{\xi}}^{2}-\tilde{\boldsymbol{\xi}}^{1}\right)
$$

and then

$$
\left\|\tilde{\boldsymbol{\xi}}^{2}-\tilde{\boldsymbol{\xi}}^{1}\right\| \leq\left\|\boldsymbol{w}^{1}\right\|_{L^{\infty}}\left\|\tilde{T}^{2}-\tilde{T}^{1}\right\|+\left\|\tilde{T}^{2}\left(\boldsymbol{w}^{1}-\boldsymbol{w}^{2}\right)\right\| .
$$

By this last estimate and (3.18), we get

$$
\left\|\tilde{\boldsymbol{\xi}}^{2}-\tilde{\boldsymbol{\xi}}^{1}\right\| \leq C h\left\|\boldsymbol{w}^{1}\right\|_{L^{\infty}}\left\|\tilde{\boldsymbol{\xi}}^{2}-\tilde{\boldsymbol{\xi}}^{1}\right\|+\left(C\left\|\boldsymbol{w}^{1}\right\|_{L^{\infty}}+1\right)\left\|\tilde{T}^{2}\left(\boldsymbol{w}^{1}-\boldsymbol{w}^{2}\right)\right\| .
$$

Hence, for $h$ small enough,

$$
\left\|\tilde{\boldsymbol{\xi}}^{2}-\tilde{\boldsymbol{\xi}}^{1}\right\| \leq\left(C\left\|\boldsymbol{w}^{1}\right\|_{L^{\infty}}+1\right)\left\|\tilde{T}^{2}\left(\boldsymbol{w}^{1}-\boldsymbol{w}^{2}\right)\right\|,
$$

which gives, using the fact that $\left\|\boldsymbol{w}^{1}\right\|_{L^{\infty}} \leq C$,

$$
\left\|\tilde{\boldsymbol{\xi}}^{2}-\tilde{\boldsymbol{\xi}}^{1}\right\| \leq C\left\|\tilde{T}^{2}\left(\boldsymbol{w}^{1}-\boldsymbol{w}^{2}\right)\right\|
$$

Hence, from (3.18), (3.19) and $\operatorname{div}\left(\tilde{\boldsymbol{\xi}}^{2}-\tilde{\boldsymbol{\xi}}^{1}\right)=0$,

$$
\left\|\tilde{\boldsymbol{\xi}}^{2}-\tilde{\boldsymbol{\xi}}^{1}\right\|_{X}+\left\|\tilde{T}^{2}-\tilde{T}^{1}\right\| \leq C\left\|\tilde{T}^{2}\left(\boldsymbol{w}^{1}-\boldsymbol{w}^{2}\right)\right\| .
$$

On the other hand, using Lemma 6 and the inverse inequality, we get

$$
\begin{aligned}
\left\|\tilde{T}^{2}\left(\boldsymbol{w}^{1}-\boldsymbol{w}^{2}\right)\right\| & \leq\left\|\tilde{T}^{2}\right\|_{L^{\infty}}\left\|\boldsymbol{w}^{1}-\boldsymbol{w}^{2}\right\| \\
& \leq C h^{-1}\left\|\tilde{T}^{2}\right\|\left\|\boldsymbol{w}^{1}-\boldsymbol{w}^{2}\right\| \\
& \leq C h^{-1}\|Q\|\left\|\boldsymbol{w}^{1}-\boldsymbol{w}^{2}\right\| .
\end{aligned}
$$

Consequently,

$$
\left\|F\left({\underset{\sim}{\boldsymbol{w}^{1}}}^{1}\right)-F\left({\underset{\sim}{\boldsymbol{w}}}^{2}\right)\right\| \leq C h^{-1}\|Q\|\left\|{\underset{\sim}{\boldsymbol{w}}}^{1}-{\underset{\sim}{\boldsymbol{w}}}^{2}\right\|
$$

Replacing, in (3.10), $(\underset{\sim}{\boldsymbol{\tau}}, \underset{\sim}{\boldsymbol{v}})$ by $\left({\underset{\sim}{\boldsymbol{\sigma}}}^{1},{\underset{\sim}{\boldsymbol{u}}}^{1}\right)$, we get

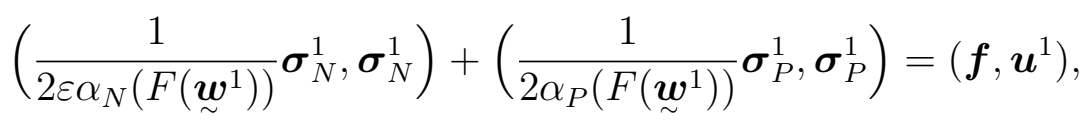


and then

$$
\frac{1}{2 \varepsilon \alpha_{N, \max }}\left\|\boldsymbol{\sigma}_{N}^{1}\right\|^{2}+\frac{1}{2 \alpha_{P, \max }}\left\|\boldsymbol{\sigma}_{P}^{1}\right\|^{2} \leq\|\boldsymbol{f}\|\left\|\boldsymbol{u}^{1}\right\| .
$$

Now, due to the discrete inf-sup condition (see Farhloul and Fortin [11]), we get

$$
\beta^{*}\left\|{\underset{\boldsymbol{u}}{1}}^{1}\right\| \leq \sup _{\underset{\sim}{\boldsymbol{\tau} \in \Sigma_{h}}} \frac{\left(\operatorname{div}\left(\boldsymbol{\tau}_{N}+\boldsymbol{\tau}_{P}-q I\right), \boldsymbol{u}^{1}\right)+\left(\operatorname{as}\left(\boldsymbol{\tau}_{N}\right), \omega^{1}\right)}{\|\underset{\sim}{\boldsymbol{\tau}}\|_{\Sigma}}
$$

and thanks to (3.10), we obtain

$$
\beta^{*}\left\|{\underset{\sim}{\boldsymbol{u}}}^{1}\right\| \leq \sup _{\boldsymbol{\sim} \in \Sigma_{h}} \frac{-\left(\frac{1}{2 \varepsilon \alpha_{N}\left(F\left({\underset{\sim}{\boldsymbol{w}}}^{1}\right)\right)} \boldsymbol{\sigma}_{N}^{1}, \boldsymbol{\tau}_{N}\right)-\left(\frac{1}{2 \alpha_{P}\left(F\left({\underset{\sim}{\boldsymbol{w}}}^{1}\right)\right)} \boldsymbol{\sigma}_{P}^{1}, \boldsymbol{\tau}_{P}\right)}{\|\underset{\sim}{\boldsymbol{\tau}}\|_{\Sigma}} .
$$

Finally, we have

$$
\beta^{*}\left\|{\underset{\sim}{\boldsymbol{u}}}^{1}\right\| \leq \frac{1}{2 \varepsilon \alpha_{N, \min }}\left\|\boldsymbol{\sigma}_{N}^{1}\right\|+\frac{1}{2 \alpha_{P, \min }}\left\|\boldsymbol{\sigma}_{P}^{1}\right\|
$$

which implies

$$
\left\|\boldsymbol{u}^{1}\right\| \leq C\left(\left\|\boldsymbol{\sigma}_{N}^{1}\right\|+\left\|\boldsymbol{\sigma}_{P}^{1}\right\|\right)
$$

and from (3.21), we get

$$
\left\|\boldsymbol{\sigma}_{N}^{1}\right\|+\left\|\boldsymbol{\sigma}_{P}^{1}\right\| \leq C\|\boldsymbol{f}\|
$$

Thus, from (3.14) and (3.20), there exists a constant $C$ depending on $h$ such that

$$
\left\|\boldsymbol{\sigma}_{N}^{2}-\boldsymbol{\sigma}_{N}^{1}\right\|^{2}+\left\|\boldsymbol{\sigma}_{P}^{2}-\boldsymbol{\sigma}_{P}^{1}\right\|^{2} \leq C(h)\left\|\boldsymbol{w}^{2}-{\underset{\sim}{\boldsymbol{w}}}^{1}\right\|\left(\left\|\boldsymbol{\sigma}_{N}^{2}-\boldsymbol{\sigma}_{N}^{1}\right\|+\left\|\boldsymbol{\sigma}_{P}^{2}-\boldsymbol{\sigma}_{P}^{1}\right\|\right) .
$$

Then,

$$
\left(\left\|\boldsymbol{\sigma}_{N}^{2}-\boldsymbol{\sigma}_{N}^{1}\right\|^{2}+\left\|\boldsymbol{\sigma}_{P}^{2}-\boldsymbol{\sigma}_{P}^{1}\right\|^{2}\right)^{1 / 2} \leq C(h)\left\|\underset{\sim}{\boldsymbol{w}^{2}}-{\underset{\sim}{\boldsymbol{w}}}^{1}\right\|
$$

Now, let $V_{h}$ be the following discrete kernel:

$$
V_{h}=\left\{\underset{\sim}{\boldsymbol{\tau}} \in \Sigma_{h} ;\left(\operatorname{div}\left(\boldsymbol{\tau}_{N}+\boldsymbol{\tau}_{P}-q I\right), \boldsymbol{v}\right)+\left(a s\left(\boldsymbol{\tau}_{N}\right), \theta\right)=0, \forall \underset{\sim}{\boldsymbol{v}}=(\boldsymbol{v}, \theta) \in M_{h}\right\}
$$

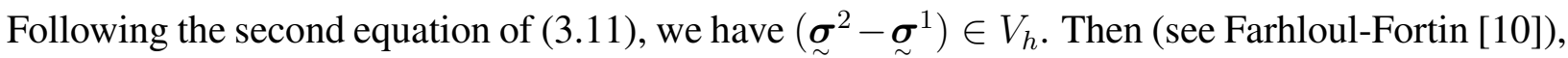

$$
\left\|p^{2}-p^{1}\right\| \leq C\left(\left\|\boldsymbol{\sigma}_{N}^{2}-\boldsymbol{\sigma}_{N}^{1}\right\|+\left\|\boldsymbol{\sigma}_{P}^{2}-\boldsymbol{\sigma}_{P}^{1}\right\|\right)
$$

On the other hand, following the second equation of (3.11) again, we obtain

$$
\left(\operatorname{div}\left[\left(\boldsymbol{\sigma}_{N}^{2}+\boldsymbol{\sigma}_{P}^{2}-p^{2} I\right)-\left(\boldsymbol{\sigma}_{N}^{1}+\boldsymbol{\sigma}_{P}^{1}-p^{1} I\right)\right], \boldsymbol{v}\right)=0, \forall \boldsymbol{v} \in\left(\Pi_{K \in \mathcal{T}_{h}} P_{0}(K)\right)^{2},
$$


and then

$$
\operatorname{div}\left[\left(\boldsymbol{\sigma}_{N}^{2}+\boldsymbol{\sigma}_{P}^{2}-p^{2} I\right)-\left(\boldsymbol{\sigma}_{N}^{1}+\boldsymbol{\sigma}_{P}^{1}-p^{1} I\right)\right]=0 .
$$

Consequently, by (3.22) and (3.23), we get

$$
\left\|{\underset{\sim}{\boldsymbol{\sigma}}}^{2}-{\underset{\sim}{\boldsymbol{\sigma}}}^{1}\right\|_{\Sigma} \leq C(h)\left\|{\underset{\sim}{\boldsymbol{w}}}^{2}-{\underset{\sim}{\boldsymbol{w}}}^{1}\right\|
$$

It remains to estimate $\left\|\underset{\sim}{\boldsymbol{u}}{ }^{2}-\underset{\sim}{\boldsymbol{u}}{ }^{1}\right\|$. To this end, we use, once again, the discrete inf-sup condition. From the first equation of (3.12), we obtain

$$
\begin{aligned}
& \beta^{*}\left\|{\underset{\sim}{\boldsymbol{u}}}^{2}-{\underset{\sim}{\boldsymbol{u}}}^{1}\right\| \leq \sup _{\boldsymbol{\tau} \in \Sigma_{h}} \frac{\left(\operatorname{div}\left(\boldsymbol{\tau}_{N}+\boldsymbol{\tau}_{P}-q I\right), \boldsymbol{u}^{2}-\boldsymbol{u}^{1}\right)+\left(\operatorname{as}\left(\boldsymbol{\tau}_{N}\right), \omega^{2}-\omega^{1}\right)}{\|\underset{\sim}{\boldsymbol{\tau}}\|_{\Sigma}} \\
& \leq \quad \sup _{\underset{\sim}{\boldsymbol{\tau}} \in \Sigma_{h}} \frac{1}{\|\underset{\sim}{\boldsymbol{\tau}}\|_{\Sigma}}\left\{\left(\left(\frac{1}{2 \varepsilon \alpha_{N}\left(F\left({\underset{\sim}{\boldsymbol{w}}}^{1}\right)\right)}-\frac{1}{2 \varepsilon \alpha_{N}\left(F\left({\underset{\sim}{\boldsymbol{w}}}^{2}\right)\right)}\right) \boldsymbol{\sigma}_{N}^{1}, \boldsymbol{\tau}_{N}\right)\right. \\
& +\left(\left(\frac{1}{2 \alpha_{P}\left(F\left({\underset{\sim}{\boldsymbol{w}}}^{1}\right)\right)}-\frac{1}{2 \alpha_{P}\left(F\left({\underset{\sim}{\boldsymbol{w}}}^{2}\right)\right)}\right) \boldsymbol{\sigma}_{P}^{1}, \boldsymbol{\tau}_{P}\right) \\
& +\left(\frac{1}{2 \varepsilon \alpha_{N}\left(F\left({\underset{\sim}{w}}^{2}\right)\right)}\left(\boldsymbol{\sigma}_{N}^{1}-\boldsymbol{\sigma}_{N}^{2}\right), \boldsymbol{\tau}_{N}\right) \\
& \left.+\left(\frac{1}{2 \alpha_{P}\left(F\left({\underset{\sim}{\boldsymbol{w}}}^{2}\right)\right)}\left(\boldsymbol{\sigma}_{P}^{1}-\boldsymbol{\sigma}_{P}^{2}\right), \boldsymbol{\tau}_{P}\right)\right\}
\end{aligned}
$$

and then

$$
\begin{aligned}
\beta^{*}\left\|{\underset{\sim}{\boldsymbol{u}}}^{2}-{\underset{\sim}{\boldsymbol{u}}}^{1}\right\| & \leq \frac{1}{2 \varepsilon}\left\|\frac{1}{\alpha_{N}\left(F\left({\underset{\sim}{\boldsymbol{w}}}^{1}\right)\right)}-\frac{1}{\alpha_{N}\left(F\left({\underset{\sim}{\boldsymbol{w}}}^{2}\right)\right)}\right\|\left\|\boldsymbol{\sigma}_{N}^{1}\right\|_{L^{\infty}} \\
& +\frac{1}{2}\left\|\frac{1}{\alpha_{P}\left(F\left({\underset{\sim}{\boldsymbol{w}}}^{1}\right)\right.}-\frac{1}{\alpha_{P}\left(F\left({\underset{\sim}{\boldsymbol{w}}}^{2}\right)\right.}\right\|\left\|\boldsymbol{\sigma}_{P}^{1}\right\|_{L^{\infty}} \\
& +\frac{1}{2 \varepsilon \alpha_{N, \min }}\left\|\boldsymbol{\sigma}_{N}^{2}-\boldsymbol{\sigma}_{N}^{1}\right\|+\frac{1}{2 \alpha_{P, \min }}\left\|\boldsymbol{\sigma}_{P}^{2}-\boldsymbol{\sigma}_{P}^{1}\right\| .
\end{aligned}
$$

Thus,

$$
\beta^{*}\left\|{\underset{\sim}{\boldsymbol{u}}}^{2}-{\underset{\sim}{\boldsymbol{u}}}^{1}\right\| \leq C\left(h^{-1}\left(\left\|\boldsymbol{\sigma}_{N}^{1}\right\|+\left\|\boldsymbol{\sigma}_{P}^{1}\right\|\right)\left\|F\left({\underset{\sim}{\boldsymbol{w}}}^{2}\right)-F\left({\underset{\sim}{\boldsymbol{w}}}^{1}\right)\right\|+\left\|\boldsymbol{\sigma}_{N}^{2}-\boldsymbol{\sigma}_{N}^{1}\right\|+\left\|\boldsymbol{\sigma}_{P}^{2}-\boldsymbol{\sigma}_{P}^{1}\right\|\right)
$$

and, by (3.20) and (3.24), we get

$$
\left\|{\underset{\sim}{\boldsymbol{u}}}^{2}-{\underset{\sim}{\boldsymbol{u}}}^{1}\right\| \leq C(h)\left\|{\underset{\sim}{\boldsymbol{w}}}^{2}-{\underset{\sim}{\boldsymbol{w}}}^{1}\right\|
$$

Finally, from (3.24) and (3.25), we get (3.9).

It is clear that (3.9) implies the continuity of $\mathcal{D}$. On the other hand, by (3.9) and the fact that $\Sigma_{h} \times M_{h}$ is a finite-dimensional space, $\mathcal{D}$ is a compact mapping. 


\subsubsection{Proof of (H2)}

Now we prove that there exists a constant $C>0$ such that, for all $\lambda \in[0,1]$ and $\underset{\sim}{\boldsymbol{r}}, \underset{\sim}{\boldsymbol{w}}) \in \Sigma_{h} \times M_{h}$, if $(\underset{\sim}{\boldsymbol{r}}, \underset{\sim}{\boldsymbol{w}})=\lambda \mathcal{D}(\underset{\sim}{\boldsymbol{r}}, \underset{\sim}{\boldsymbol{w}})$ then $(\underset{\sim}{\boldsymbol{r}}, \underset{\sim}{\boldsymbol{w}})$ satisfies $\|(\underset{\sim}{\boldsymbol{r}}, \underset{\sim}{\boldsymbol{w}})\|_{\Sigma \times \tilde{M}} \leq C$, where $\tilde{M}=\left[L^{2}(\Omega)\right]^{2} \times L^{2}(\Omega)$. Let $\lambda \in[0,1]$ and $(\underset{\sim}{\boldsymbol{r}}, \underset{\sim}{\boldsymbol{w}})=\left(\left(\boldsymbol{r}_{N}, \boldsymbol{r}_{P}, t\right),(\boldsymbol{w}, s)\right) \in \Sigma_{h} \times M_{h}$ such that $\lambda \mathcal{D}(\underset{\sim}{\boldsymbol{r}}, \underset{\sim}{\boldsymbol{w}})=(\underset{\sim}{\boldsymbol{r}}, \underset{\sim}{\boldsymbol{w}})$.

- If $\lambda=0$, then $\lambda \mathcal{D}(\underset{\sim}{\boldsymbol{r}}, \underset{\sim}{\boldsymbol{w}})=(0,0)=(\underset{\sim}{\boldsymbol{r}}, \underset{\sim}{\boldsymbol{w}})$ which means that $\underset{\sim}{\boldsymbol{r}}=0$ and $\underset{\sim}{\boldsymbol{w}}=0$.

- Suppose now $\lambda \in] 0,1]$. Then

$$
\lambda \mathcal{D}(\underset{\sim}{\boldsymbol{r}}, \underset{\sim}{\boldsymbol{w}})=(\underset{\sim}{\boldsymbol{r}}, \underset{\sim}{\boldsymbol{w}}) \Longrightarrow \mathcal{D}(\underset{\sim}{\boldsymbol{r}}, \underset{\sim}{\boldsymbol{w}})=\left(\frac{1}{\lambda} \underset{\sim}{\boldsymbol{r}}, \frac{1}{\lambda} \underset{\sim}{\boldsymbol{w}}\right)
$$

Hence, $\forall \underset{\sim}{\boldsymbol{\tau}}=\left(\boldsymbol{\tau}_{N}, \boldsymbol{\tau}_{P}, q\right) \in \Sigma_{h}$ and $\forall \underset{\sim}{\boldsymbol{v}}=(\boldsymbol{v}, \theta) \in M_{h}$,

$$
\left\{\begin{array}{l}
\left(\frac{1}{2 \varepsilon \alpha_{N}(F(\underset{\sim}{\boldsymbol{w}}))} \frac{\boldsymbol{r}_{N}}{\lambda}, \boldsymbol{\tau}_{N}\right)+\left(\frac{1}{2 \alpha_{P}(F(\underset{\sim}{\boldsymbol{w}}))} \frac{\boldsymbol{r}_{P}}{\lambda}, \boldsymbol{\tau}_{P}\right)+\left(\operatorname{div}\left(\boldsymbol{\tau}_{N}+\boldsymbol{\tau}_{P}-q I\right), \frac{\boldsymbol{w}}{\lambda}\right)+ \\
\left(\operatorname{as}\left(\boldsymbol{\tau}_{N}\right), \frac{s}{\lambda}\right)=0 \\
\left(\frac{1}{\lambda} \operatorname{div}\left(\boldsymbol{r}_{N}+\boldsymbol{r}_{P}-t I\right), \boldsymbol{v}\right)+\left(\frac{1}{\lambda} \operatorname{as}\left(\boldsymbol{r}_{N}\right), \theta\right)+(\boldsymbol{f}, \boldsymbol{v})=0,
\end{array}\right.
$$

and then

$$
\left\{\begin{array}{l}
\left(\frac{1}{2 \varepsilon \alpha_{N}(F(\underset{\sim}{\boldsymbol{w}}))} \boldsymbol{r}_{N}, \boldsymbol{\tau}_{N}\right)+\left(\frac{1}{2 \alpha_{P}(F(\underset{\sim}{\boldsymbol{w}}))} \boldsymbol{r}_{P}, \boldsymbol{\tau}_{P}\right)+\left(\operatorname{div}\left(\boldsymbol{\tau}_{N}+\boldsymbol{\tau}_{P}-q I\right), \boldsymbol{w}\right)+ \\
\left(a s\left(\boldsymbol{\tau}_{N}\right), s\right)=0 \\
\left(\operatorname{div}\left(\boldsymbol{r}_{N}+\boldsymbol{r}_{P}-t I\right), \boldsymbol{v}\right)+\left(a s\left(r_{N}\right), \theta\right)+\lambda(\boldsymbol{f}, \boldsymbol{v})=0 .
\end{array}\right.
$$

Choosing $\underset{\sim}{\boldsymbol{\tau}}=\underset{\sim}{\boldsymbol{r}}$ and $\underset{\sim}{\boldsymbol{v}}=\underset{\sim}{\boldsymbol{w}}$ in (3.26), we get

$$
\left(\frac{1}{2 \varepsilon \alpha_{N}(F(\underset{\sim}{\boldsymbol{w}}))} \boldsymbol{r}_{N}, \boldsymbol{r}_{N}\right)+\left(\frac{1}{2 \alpha_{P}(F(\underset{\sim}{\boldsymbol{w}}))} \boldsymbol{r}_{P}, \boldsymbol{r}_{P}\right)=\lambda(\boldsymbol{f}, \boldsymbol{w})
$$

and then

$$
\frac{1}{2 \varepsilon \alpha_{N, \max }}\left\|\boldsymbol{r}_{N}\right\|^{2}+\frac{1}{2 \alpha_{P, \max }}\left\|\boldsymbol{r}_{P}\right\|^{2} \leq \lambda\|\boldsymbol{f}\|\|\boldsymbol{w}\| .
$$

which gives

$$
\left\|\boldsymbol{r}_{N}\right\|^{2}+\left\|\boldsymbol{r}_{P}\right\|^{2} \leq C\|\boldsymbol{f}\|\|\boldsymbol{w}\|
$$

On the other hand, using the discrete inf-sup condition and the first equation of (3.26), we get 


$$
\begin{aligned}
& \beta^{*}\|\underset{\sim}{\boldsymbol{w}}\| \leq \sup _{\underset{\sim}{\boldsymbol{\tau} \in \Sigma_{h}}} \frac{\left(\operatorname{div}\left(\boldsymbol{\tau}_{N}+\boldsymbol{\tau}_{P}-q I\right), \boldsymbol{w}\right)+\left(\operatorname{as}\left(\boldsymbol{\tau}_{N}\right), s\right)}{\|\underset{\sim}{\boldsymbol{\tau}}\|_{\Sigma}} \\
& -\left(\frac{1}{2 \varepsilon \alpha_{N}(F(\underset{\sim}{\boldsymbol{w}}))} \boldsymbol{r}_{N}, \boldsymbol{\tau}_{N}\right)-\left(\frac{1}{2 \alpha_{P}(F(\underset{\sim}{\boldsymbol{w}}))} \boldsymbol{r}_{P}, \boldsymbol{\tau}_{P}\right) \\
& =\sup _{\underset{\sim}{\boldsymbol{\tau}} \in \Sigma_{h}} \quad\|\underset{\sim}{\boldsymbol{\tau}}\|_{\Sigma} \\
& \leq \frac{1}{2 \varepsilon \alpha_{N, \min }}\left\|\boldsymbol{r}_{N}\right\|+\frac{1}{2 \alpha_{P, \min }}\left\|\boldsymbol{r}_{P}\right\| \text {. }
\end{aligned}
$$

Thus,

$$
\|\underset{\sim}{\boldsymbol{w}}\| \leq C\left(\left\|\boldsymbol{r}_{N}\right\|+\left\|\boldsymbol{r}_{P}\right\|\right)
$$

Therefore, we get, from (3.27) and (3.28)

$$
\left(\left\|\boldsymbol{r}_{N}\right\|^{2}+\left\|\boldsymbol{r}_{P}\right\|^{2}\right)^{1 / 2} \leq C\|\boldsymbol{f}\|
$$

Following (3.28) and (3.29), we obtain also

$$
\|\underset{\sim}{\boldsymbol{w}}\| \leq C\|\boldsymbol{f}\|
$$

On the other hand, from (3.26), we get

$$
\left(\operatorname{div}\left(\boldsymbol{r}_{N}+\boldsymbol{r}_{P}-t I\right), \boldsymbol{v}\right)=-\lambda(\boldsymbol{f}, \boldsymbol{v}), \forall \boldsymbol{v} \in\left(\Pi_{K \in \mathcal{T}_{h}} P_{0}(K)\right)^{2},
$$

and,

$$
\left\|\operatorname{div}\left(\boldsymbol{r}_{N}+\boldsymbol{r}_{P}-t I\right)\right\| \leq \lambda\|\boldsymbol{f}\| .
$$

It remains to estimate $\|t\|$. From (3.26), we have

$$
\left(\operatorname{div}\left(\boldsymbol{r}_{N}+\boldsymbol{r}_{P}-t I\right), \boldsymbol{v}\right)+\lambda(\boldsymbol{f}, \boldsymbol{v})=0, \forall \boldsymbol{v} \in\left(\Pi_{K \in \mathcal{T}_{h}} P_{0}(K)\right)^{2},
$$

and then

$$
\left(\operatorname{div}\left(\boldsymbol{r}_{N}+\boldsymbol{r}_{P}-t I\right), \boldsymbol{v}\right)+\lambda\left(P_{h}^{0} \boldsymbol{f}, \boldsymbol{v}\right)=0, \forall \boldsymbol{v} \in\left(\Pi_{K \in \mathcal{T}_{h}} P_{0}(K)\right)^{2}
$$

where $P_{h}^{0}$ stands for the $L^{2}$-projection operator on $\left(\Pi_{K \in \mathcal{T}_{h}} P_{0}(K)\right)^{2}$.

Let $\tau^{*}$, such that

$$
\left\{\begin{array}{l}
\boldsymbol{\tau}^{*} \in\left\{\boldsymbol{\tau} \in[H(\operatorname{div} ; \Omega)]^{2} ; \boldsymbol{\tau}_{\mid K} \in\left(R T_{0}(K)\right)^{2}, \forall K \in \mathcal{T}_{h}\right\} \\
\operatorname{div} \boldsymbol{\tau}^{*}=P_{h}^{0} \boldsymbol{f} \\
\left\|\boldsymbol{\tau}^{*}\right\|_{H(\operatorname{div} ; \Omega)} \leq C\left\|P_{h}^{0} \boldsymbol{f}\right\| \leq C\|\boldsymbol{f}\|
\end{array}\right.
$$


Then, following (3.32), we get

$$
\left(\operatorname{div}\left(\boldsymbol{r}_{N}+\boldsymbol{r}_{P}+\lambda \boldsymbol{\tau}^{*}-t I\right), \boldsymbol{v}\right)=0, \forall \boldsymbol{v} \in\left(\Pi_{K \in \mathcal{T}_{h}} P_{0}(K)\right)^{2} .
$$

Therefore, following Farhloul-Fortin [10], we get

$$
\|t\| \leq\left\|\boldsymbol{r}_{N}+\boldsymbol{r}_{P}+\lambda \boldsymbol{\tau}^{*}\right\| \leq C\left(\left\|\boldsymbol{r}_{N}\right\|+\left\|\boldsymbol{r}_{P}\right\|+\lambda\left\|\boldsymbol{\tau}^{*}\right\|\right),
$$

and, from (3.29) and (3.33),

$$
\|t\| \leq C\|\boldsymbol{f}\|
$$

Then, from (3.29), (3.30), (3.31) and (3.34), we obtain the desired result:

$$
\|(\underset{\sim}{\boldsymbol{r}}, \underset{\sim}{\boldsymbol{w}})\|_{\Sigma \times \tilde{M}} \leq C\|\boldsymbol{f}\|
$$

Finally, the hypotheses of Schaefer's Fixed Point Theorem are satisfied. Problem (3.7) has at least one solution. Then the discrete problem (3.1) has also at least one solution.

\section{Error estimates}

To get the error estimates, we first need the following result:

Proposition 8. Let $r>2$, then there exists an operator

$$
\begin{aligned}
\Pi_{h}: & \Sigma \cap\left(\left[L^{r}(\Omega)\right]^{2 \times 2} \times\left[L^{r}(\Omega)\right]_{s}^{2 \times 2} \times L^{r}(\Omega)\right) \longrightarrow \Sigma_{h}, \\
& \boldsymbol{\tau}=\left(\boldsymbol{\tau}_{N}, \boldsymbol{\tau}_{P}, q\right) \longmapsto \Pi_{h}\left(\boldsymbol{\tau}_{N}, \boldsymbol{\tau}_{P}, q\right)=\boldsymbol{\tau}_{\sim}=\left(\boldsymbol{\tau}_{N h}, \boldsymbol{\tau}_{P h}, q_{h}\right)
\end{aligned}
$$

such that, $\forall \underset{\sim}{\boldsymbol{v}}=(\boldsymbol{v}, \theta) \in M_{h}$,

$$
\left(\operatorname{div}\left[\left(\boldsymbol{\tau}_{N}+\boldsymbol{\tau}_{P}-q I\right)-\left(\boldsymbol{\tau}_{N h}+\boldsymbol{\tau}_{P h}-q_{h} I\right)\right], \boldsymbol{v}\right)+\left(a s\left(\boldsymbol{\tau}_{N}-\boldsymbol{\tau}_{N h}\right), \theta\right)=0 .
$$

Moreover, there exists a constant $C$ independent of $h$, such that, for all $\boldsymbol{\tau}_{N} \in\left[H^{1}(\Omega)\right]^{2 \times 2}, \boldsymbol{\tau}_{P} \in$ $\left[H^{1}(\Omega)\right]_{s}^{2 \times 2}$ and $q \in H^{1}(\Omega) \cap L_{0}^{2}(\Omega)$,

$$
\left\|\left(\boldsymbol{\tau}_{N}, \boldsymbol{\tau}_{P}, q\right)-\Pi_{h}\left(\boldsymbol{\tau}_{N}, \boldsymbol{\tau}_{P}, q\right)\right\| \leq C h\left(\left|\boldsymbol{\tau}_{N}\right|_{1, \Omega}+\left|\boldsymbol{\tau}_{P}\right|_{1, \Omega}+|q|_{1, \Omega}\right) .
$$

Proof. The proof of this proposition is similar to proposition 3.3 in Farhloul-Zine [12]. In the present case (see Farhloul-Fortin [11]), one uses the stability of the Mini-element $P_{1}^{+}-P_{1}$ for the Stokes problem. 
We are now in a position to establish optimal error estimates for problem (3.1). In the following $(\underset{\sim}{\boldsymbol{\sigma}}, \underset{\sim}{\boldsymbol{u}}, \boldsymbol{\xi}, T)$ denotes a solution of problem $(2.1)$ and $\left.\underset{\sim}{\boldsymbol{\sigma}_{h}}, \underset{\sim}{\boldsymbol{u}_{h}}, \boldsymbol{\xi}_{h}, T_{h}\right)$ denotes a solution of problem (3.1).

We shall use the following notations:

$\|\underset{\sim}{\boldsymbol{\sigma}}\|_{m, \Omega}=\left(\left\|\boldsymbol{\sigma}_{N}\right\|_{m, \Omega}^{2}+\left\|\boldsymbol{\sigma}_{P}\right\|_{m, \Omega}^{2}+\|p\|_{m, \Omega}^{2}\right)^{1 / 2}$ and $\|\underset{\sim}{\boldsymbol{v}}\|_{m, \Omega}=\left(\|\boldsymbol{v}\|_{m, \Omega}^{2}+\|\theta\|_{m, \Omega}^{2}\right)^{1 / 2}$.

Theorem 9. Suppose that $\underset{\sim}{\boldsymbol{\sigma}} \in\left[H^{1}(\Omega) \cap L^{\infty}(\Omega)\right]^{2 \times 2} \times\left[H^{1}(\Omega) \cap L^{\infty}(\Omega)\right]_{s}^{2 \times 2} \times H^{1}(\Omega), \underset{\sim}{\boldsymbol{u}} \in$ $\left[H^{1}(\Omega)\right]^{2} \times H^{1}(\Omega), \boldsymbol{\xi} \in\left[H^{1}(\Omega)\right]^{2}$ and $T \in H^{1}(\Omega) \cap L^{\infty}(\Omega)$ such that

$$
\max \left\{\|\underset{\sim}{\boldsymbol{\sigma}}\|_{1, \Omega},\|\underset{\sim}{\boldsymbol{u}}\|_{1, \Omega},\|\boldsymbol{\xi}\|_{1, \Omega},\|T\|_{1, \Omega},\left\|\boldsymbol{\sigma}_{N}\right\|_{L^{\infty}},\left\|\boldsymbol{\sigma}_{P}\right\|_{L^{\infty}},\|T\|_{L^{\infty}}\right\} \leq R
$$

with $R$ small enough. Then, there exists a positive constant $C$ independent of $h$ such that

$$
\left\|\underset{\sim}{\boldsymbol{\sigma}}-\boldsymbol{\sigma}_{h}\right\| \leq C h,\left\|\underset{\sim}{\boldsymbol{u}}-\underset{\sim}{\boldsymbol{u}_{h}}\right\| \leq C h,\left\|\boldsymbol{\xi}-\boldsymbol{\xi}_{h}\right\| \leq C h \text { and }\left\|T-T_{h}\right\| \leq C h .
$$

Proof. Following (2.1) and (3.1), we have

$$
\begin{aligned}
& \left\{\begin{array}{l}
\left(\frac{1}{2 \varepsilon \alpha_{N}(T)} \boldsymbol{\sigma}_{N}-\frac{1}{2 \varepsilon \alpha_{N}\left(T_{h}\right)} \boldsymbol{\sigma}_{N h}, \boldsymbol{\tau}_{N}\right)+\left(\frac{1}{2 \alpha_{P}(T)} \boldsymbol{\sigma}_{P}-\frac{1}{2 \alpha_{P}\left(T_{h}\right)} \boldsymbol{\sigma}_{P h}, \boldsymbol{\tau}_{P}\right)+ \\
\left(\operatorname{div}\left(\boldsymbol{\tau}_{N}+\boldsymbol{\tau}_{P}-q I\right), \boldsymbol{u}-\boldsymbol{u}_{h}\right)+\left(a s\left(\boldsymbol{\tau}_{N}\right), \omega-\omega_{h}\right)=0, \forall \underset{\sim}{\boldsymbol{\tau}} \in \Sigma_{h},
\end{array}\right. \\
& \left\{\begin{array}{l}
\left(\operatorname{div}\left[\left(\boldsymbol{\sigma}_{N}+\boldsymbol{\sigma}_{P}-p I\right)-\left(\boldsymbol{\sigma}_{N h}+\boldsymbol{\sigma}_{P h}-p_{h} I\right)\right], \boldsymbol{v}\right)+ \\
\left(a s\left(\boldsymbol{\sigma}_{N}-\boldsymbol{\sigma}_{N h}\right), \theta\right)=0, \forall \underset{\sim}{\boldsymbol{v}}=(\boldsymbol{v}, \theta) \in M_{h}
\end{array}\right. \\
& \frac{1}{\kappa}\left(\boldsymbol{\xi}-\boldsymbol{\xi}_{h}, \boldsymbol{\eta}\right)+\left(\operatorname{div} \boldsymbol{\eta}, T-T_{h}\right)+\frac{1}{\kappa}\left(T \boldsymbol{u}-T_{h} \boldsymbol{u}_{h}, \boldsymbol{\eta}\right)=0, \forall \boldsymbol{\eta} \in X_{h}, \\
& \left(\operatorname{div}\left(\boldsymbol{\xi}-\boldsymbol{\xi}_{h}\right), \psi\right)=0, \quad \forall \psi \in Y_{h} .
\end{aligned}
$$

Let

- $\boldsymbol{\sigma}_{\sim^{*}}{ }^{*}=\left(\boldsymbol{\sigma}_{N h}^{*}, \boldsymbol{\sigma}_{P h}^{*}, p_{h}^{*}\right)=\Pi_{h}\left(\boldsymbol{\sigma}_{N}, \boldsymbol{\sigma}_{P}, p\right)$,

- ${\underset{\sim}{\boldsymbol{u}_{h}}}^{*}=\left(\boldsymbol{u}_{h}^{*}, \omega_{h}^{*}\right)=\left(P_{h}^{0} \boldsymbol{u}, I_{C l} \omega\right)$ the interpolate of $(\boldsymbol{u}, \omega)$ in $M_{h}$,

- $\boldsymbol{\xi}_{h}^{*}=R T_{0}(\boldsymbol{\xi})$, the Raviart-Thomas interpolate,

- $T_{h}^{*}=\rho_{h}^{0} T$, the interpolate of $T$ in $Y_{h}$, 
where $I_{C l}$ denotes the Clément interpolation operator (cf. [5]).

Now from (4.1) and (4.4), we get

$$
\left\{\begin{array}{l}
\left(\operatorname{div}\left[\left(\boldsymbol{\sigma}_{N h}^{*}+\boldsymbol{\sigma}_{P h}^{*}-p_{h}^{*} I\right)-\left(\boldsymbol{\sigma}_{N h}+\boldsymbol{\sigma}_{P h}-p_{h} I\right)\right], \boldsymbol{v}\right)+ \\
\left(\operatorname{as}\left(\boldsymbol{\sigma}_{N h}^{*}-\boldsymbol{\sigma}_{N h}\right), \theta\right)=0, \forall \underset{\sim}{\boldsymbol{v}}=(\boldsymbol{v}, \theta) \in M_{h}
\end{array}\right.
$$

Choosing $\underset{\sim}{\boldsymbol{\tau}}=\boldsymbol{\sigma}_{\sim}{ }^{*}-\boldsymbol{\sigma}_{\sim}$, in (4.3), and using both (4.7) and the fact that

$$
\left(\operatorname{div}\left(\boldsymbol{\tau}_{N}+\boldsymbol{\tau}_{P}-q I\right), \boldsymbol{u}-\boldsymbol{u}_{h}^{*}\right)=0, \forall \underset{\sim}{\boldsymbol{\tau}} \in \Sigma_{h},
$$

we get

$$
\left\{\begin{array}{l}
\left(\frac{1}{2 \varepsilon \alpha_{N}(T)} \boldsymbol{\sigma}_{N}-\frac{1}{2 \varepsilon \alpha_{N}\left(T_{h}\right)} \boldsymbol{\sigma}_{N h}, \boldsymbol{\sigma}_{N h}^{*}-\boldsymbol{\sigma}_{N h}\right)+ \\
\left(\frac{1}{2 \alpha_{P}(T)} \boldsymbol{\sigma}_{P}-\frac{1}{2 \alpha_{P}\left(T_{h}\right)} \boldsymbol{\sigma}_{P h}, \boldsymbol{\sigma}_{P h}^{*}-\boldsymbol{\sigma}_{P h}\right)+\left(a s\left(\boldsymbol{\sigma}_{N h}^{*}-\boldsymbol{\sigma}_{N h}\right), \omega-\omega_{h}^{*}\right)=0 .
\end{array}\right.
$$

This is may be written as

$$
\left\{\begin{array}{l}
\left(\frac{1}{2 \varepsilon \alpha_{N}\left(T_{h}\right)}\left(\boldsymbol{\sigma}_{N h}^{*}-\boldsymbol{\sigma}_{N h}\right), \boldsymbol{\sigma}_{N h}^{*}-\boldsymbol{\sigma}_{N h}\right)+\left(\frac{1}{2 \alpha_{P}\left(T_{h}\right)}\left(\boldsymbol{\sigma}_{P h}^{*}-\boldsymbol{\sigma}_{P h}\right), \boldsymbol{\sigma}_{P h}^{*}-\boldsymbol{\sigma}_{P h}\right)= \\
\left(\frac{1}{2 \varepsilon \alpha_{N}\left(T_{h}\right)}\left(\boldsymbol{\sigma}_{N h}^{*}-\boldsymbol{\sigma}_{N}\right), \boldsymbol{\sigma}_{N h}^{*}-\boldsymbol{\sigma}_{N h}\right)+\left(\left[\frac{1}{2 \varepsilon \alpha_{N}\left(T_{h}\right)}-\frac{1}{2 \varepsilon \alpha_{N}(T)}\right] \boldsymbol{\sigma}_{N}, \boldsymbol{\sigma}_{N h}^{*}-\boldsymbol{\sigma}_{N h}\right) \\
+\left(\frac{1}{2 \alpha_{P}\left(T_{h}\right)}\left(\boldsymbol{\sigma}_{P h}^{*}-\boldsymbol{\sigma}_{P}\right), \boldsymbol{\sigma}_{P h}^{*}-\boldsymbol{\sigma}_{P h}\right)+\left(\left[\frac{1}{2 \alpha_{P}\left(T_{h}\right)}-\frac{1}{2 \alpha_{P}(T)}\right] \boldsymbol{\sigma}_{P}, \boldsymbol{\sigma}_{P h}^{*}-\boldsymbol{\sigma}_{P h}\right) \\
+\left(a s\left(\boldsymbol{\sigma}_{N h}-\boldsymbol{\sigma}_{N h}^{*}\right), \omega-\omega_{h}^{*}\right),
\end{array}\right.
$$

and then

$$
\left\{\begin{array}{l}
\frac{1}{2 \varepsilon \alpha_{N, \text { max }}}\left\|\boldsymbol{\sigma}_{N h}^{*}-\boldsymbol{\sigma}_{N h}\right\|^{2}+\frac{1}{2 \alpha_{P, \text { max }}}\left\|\boldsymbol{\sigma}_{P h}^{*}-\boldsymbol{\sigma}_{P h}\right\|^{2} \leq \\
\frac{1}{2 \varepsilon \alpha_{N, \text { min }}}\left\|\boldsymbol{\sigma}_{N h}^{*}-\boldsymbol{\sigma}_{N}\right\|\left\|\boldsymbol{\sigma}_{N h}^{*}-\boldsymbol{\sigma}_{N h}\right\|+\frac{1}{2 \varepsilon}\left\|\frac{1}{\alpha_{N}\left(T_{h}\right)}-\frac{1}{\alpha_{N}(T)}\right\|\left\|\boldsymbol{\sigma}_{N h}^{*}-\boldsymbol{\sigma}_{N h}\right\|\left\|\boldsymbol{\sigma}_{N}\right\|_{L^{\infty}} \\
+\frac{1}{2 \alpha_{P, \text { min }}}\left\|\boldsymbol{\sigma}_{P h}^{*}-\boldsymbol{\sigma}_{P}\right\|\left\|\boldsymbol{\sigma}_{P h}^{*}-\boldsymbol{\sigma}_{P h}\right\|+\frac{1}{2}\left\|\frac{1}{\alpha_{P}\left(T_{h}\right)}-\frac{1}{\alpha_{P}(T)}\right\|\left\|\boldsymbol{\sigma}_{P h}^{*}-\boldsymbol{\sigma}_{P h}\right\|\left\|\boldsymbol{\sigma}_{P}\right\|_{L^{\infty}} \\
+\left\|a s\left(\boldsymbol{\sigma}_{N h}-\boldsymbol{\sigma}_{N h}^{*}\right)\right\|\left\|\omega-\omega_{h}^{*}\right\| .
\end{array}\right.
$$


Now, using both (4.2) and the fact that

$$
\left\|\omega-\omega_{h}^{*}\right\| \leq C h|\omega|_{1, \Omega}
$$

we get

$$
\left\{\begin{array}{l}
\left\|\boldsymbol{\sigma}_{N h}^{*}-\boldsymbol{\sigma}_{N h}\right\|^{2}+\left\|\boldsymbol{\sigma}_{P h}^{*}-\boldsymbol{\sigma}_{P h}\right\|^{2} \leq C h\left(\left|\boldsymbol{\sigma}_{N}\right|_{1, \Omega}+|\omega|_{1, \Omega}\right)\left\|\boldsymbol{\sigma}_{N h}^{*}-\boldsymbol{\sigma}_{N h}\right\|+ \\
C h\left|\boldsymbol{\sigma}_{P}\right|_{1, \Omega}\left\|\boldsymbol{\sigma}_{P h}^{*}-\boldsymbol{\sigma}_{P h}\right\|+C\left\|T-T_{h}\right\|\left\|\boldsymbol{\sigma}_{N h}^{*}-\boldsymbol{\sigma}_{N h}\right\|\left\|\boldsymbol{\sigma}_{N}\right\|_{L^{\infty}}+ \\
C\left\|T-T_{h}\right\|\left\|\boldsymbol{\sigma}_{P h}^{*}-\boldsymbol{\sigma}_{P h}\right\|\left\|\boldsymbol{\sigma}_{P}\right\|_{L^{\infty}}
\end{array}\right.
$$

and then

$$
\left(\left\|\boldsymbol{\sigma}_{N h}^{*}-\boldsymbol{\sigma}_{N h}\right\|^{2}+\left\|\boldsymbol{\sigma}_{P h}^{*}-\boldsymbol{\sigma}_{P h}\right\|^{2}\right)^{1 / 2} \leq C R\left(h+\left\|T-T_{h}\right\|\right) .
$$

As

$$
\left\|T-T_{h}\right\| \leq\left\|T-T_{h}^{*}\right\|+\left\|T_{h}^{*}-T_{h}\right\| \leq\left\|T_{h}^{*}-T_{h}\right\|+C h|T|_{1, \Omega},
$$

we finally get

$$
\left\|\boldsymbol{\sigma}_{N h}^{*}-\boldsymbol{\sigma}_{N h}\right\|+\left\|\boldsymbol{\sigma}_{P h}^{*}-\boldsymbol{\sigma}_{P h}\right\| \leq C R\left(h+\left\|T_{h}^{*}-T_{h}\right\|\right) .
$$

Now, as

$$
\left(\operatorname{div}\left(\boldsymbol{\xi}-\boldsymbol{\xi}_{h}^{*}\right), \psi\right)=0, \quad \forall \psi \in Y_{h},
$$

we get, from (4.6),

$$
\operatorname{div}\left(\boldsymbol{\xi}_{h}^{*}-\boldsymbol{\xi}_{h}\right)=0 .
$$

On the other hand, from (4.5), we have

$$
\left\{\begin{array}{l}
\frac{1}{\kappa}\left(\boldsymbol{\xi}-\boldsymbol{\xi}_{h}^{*}, \boldsymbol{\eta}\right)+\frac{1}{\kappa}\left(\boldsymbol{\xi}_{h}^{*}-\boldsymbol{\xi}_{h}, \boldsymbol{\eta}\right)+\left(\operatorname{div} \boldsymbol{\eta}, T_{h}^{*}-T_{h}\right)+ \\
\frac{1}{\kappa}\left(\left(T_{h}^{*}-T_{h}\right) \boldsymbol{u}_{h}, \boldsymbol{\eta}\right)+\frac{1}{\kappa}\left(T\left(\boldsymbol{u}-\boldsymbol{u}_{h}\right), \boldsymbol{\eta}\right)+\frac{1}{\kappa}\left(\left(T-T_{h}^{*}\right) \boldsymbol{u}_{h}, \boldsymbol{\eta}\right)=0, \quad \forall \boldsymbol{\eta} \in X_{h}
\end{array}\right.
$$

and then

$$
\left\{\begin{array}{l}
\frac{1}{\kappa}\left(\boldsymbol{\xi}_{h}^{*}-\boldsymbol{\xi}_{h}, \boldsymbol{\eta}\right)+\left(\operatorname{div} \boldsymbol{\eta}, T_{h}^{*}-T_{h}\right)+\frac{1}{\kappa}\left(\left(T_{h}^{*}-T_{h}\right) \boldsymbol{u}_{h}, \boldsymbol{\eta}\right)= \\
\frac{1}{\kappa}\left(\boldsymbol{\xi}_{h}^{*}-\boldsymbol{\xi}, \boldsymbol{\eta}\right)+\frac{1}{\kappa}\left(T\left(\boldsymbol{u}_{h}-\boldsymbol{u}\right), \boldsymbol{\eta}\right)+\frac{1}{\kappa}\left(\left(T_{h}^{*}-T\right) \boldsymbol{u}_{h}, \boldsymbol{\eta}\right), \quad \forall \boldsymbol{\eta} \in X_{h} .
\end{array}\right.
$$

Therefore, using both Lemma 5 and the fact that $\operatorname{div}\left(\boldsymbol{\xi}_{h}^{*}-\boldsymbol{\xi}_{h}\right)=0$, we have

$$
\left\|T_{h}^{*}-T_{h}\right\| \leq C h\left\|\boldsymbol{\xi}_{h}^{*}-\boldsymbol{\xi}_{h}\right\|+C\left(\left\|\boldsymbol{\xi}_{h}^{*}-\boldsymbol{\xi}\right\|+\left\|T\left(\boldsymbol{u}_{h}-\boldsymbol{u}\right)\right\|+\left\|\left(T_{h}^{*}-T\right) \boldsymbol{u}_{h}\right\|\right) .
$$


On the other hand, choosing $\boldsymbol{\eta}=\boldsymbol{\xi}_{h}^{*}-\boldsymbol{\xi}_{h}$ in (4.10), and the fact that $\operatorname{div}\left(\boldsymbol{\xi}_{h}^{*}-\boldsymbol{\xi}_{h}\right)=0$, we have

$$
\left\{\begin{aligned}
\left\|\boldsymbol{\xi}_{h}^{*}-\boldsymbol{\xi}_{h}\right\|^{2}+\left(\left(T_{h}^{*}-T_{h}\right) \boldsymbol{u}_{h}, \boldsymbol{\xi}_{h}^{*}-\boldsymbol{\xi}_{h}\right)= & \left(\boldsymbol{\xi}_{h}^{*}-\boldsymbol{\xi}, \boldsymbol{\xi}_{h}^{*}-\boldsymbol{\xi}_{h}\right)+\left(T\left(\boldsymbol{u}_{h}-\boldsymbol{u}\right), \boldsymbol{\xi}_{h}^{*}-\boldsymbol{\xi}_{h}\right)+ \\
& \left(\left(T_{h}^{*}-T\right) \boldsymbol{u}_{h}, \boldsymbol{\xi}_{h}^{*}-\boldsymbol{\xi}_{h}\right) .
\end{aligned}\right.
$$

Note that, since $\left(\boldsymbol{\xi}_{h}^{*}-\boldsymbol{\xi}_{h}\right)_{\left.\right|_{K}} \in\left(R T_{0}(K)\right)^{2}, \forall K \in \mathcal{T}_{h}$, and $\operatorname{div}\left(\boldsymbol{\xi}_{h}^{*}-\boldsymbol{\xi}_{h}\right)=0$, we have

$$
\left(\boldsymbol{\xi}_{h}^{*}-\boldsymbol{\xi}_{h}\right)_{\left.\right|_{K}} \in\left(P_{0}(K)\right)^{2}, \forall K \in \mathcal{T}_{h}
$$

On the other hand, as $\boldsymbol{u}_{h_{K}} \in\left(P_{0}(K)\right)^{2}, \forall K \in \mathcal{T}_{h}$, we get

$$
\left(\left(T_{h}^{*}-T\right) \boldsymbol{u}_{h}, \boldsymbol{\xi}_{h}^{*}-\boldsymbol{\xi}_{h}\right)=\left(T_{h}^{*}-T, \boldsymbol{u}_{h} \cdot\left(\boldsymbol{\xi}_{h}^{*}-\boldsymbol{\xi}_{h}\right)\right)=0
$$

and then

$$
\left\|\boldsymbol{\xi}_{h}^{*}-\boldsymbol{\xi}_{h}\right\|^{2}=\left(\left(T_{h}-T_{h}^{*}\right) \boldsymbol{u}_{h}, \boldsymbol{\xi}_{h}^{*}-\boldsymbol{\xi}_{h}\right)+\left(\boldsymbol{\xi}_{h}^{*}-\boldsymbol{\xi}, \boldsymbol{\xi}_{h}^{*}-\boldsymbol{\xi}_{h}\right)+\left(T\left(\boldsymbol{u}_{h}-\boldsymbol{u}\right), \boldsymbol{\xi}_{h}^{*}-\boldsymbol{\xi}_{h}\right),
$$

which gives the following estimate

$$
\left\|\boldsymbol{\xi}_{h}^{*}-\boldsymbol{\xi}_{h}\right\| \leq\left\|\boldsymbol{u}_{h}\right\|_{L^{\infty}}\left\|T_{h}^{*}-T_{h}\right\|+\left\|\boldsymbol{\xi}_{h}^{*}-\boldsymbol{\xi}\right\|+\left\|T\left(\boldsymbol{u}_{h}-\boldsymbol{u}\right)\right\| .
$$

Following (4.11) and (4.12), we have

$$
\left\|T_{h}^{*}-T_{h}\right\| \leq C h\left\|\boldsymbol{u}_{h}\right\|_{L^{\infty}}\left\|T_{h}^{*}-T_{h}\right\|+C\left(\left\|\boldsymbol{\xi}_{h}^{*}-\boldsymbol{\xi}\right\|+\left\|T\left(\boldsymbol{u}_{h}-\boldsymbol{u}\right)\right\|+\left\|\left(T_{h}^{*}-T\right) \boldsymbol{u}_{h}\right\|\right),
$$

and then, for $h$ small enough, using (3.6):

$$
\left\|T_{h}^{*}-T_{h}\right\| \leq C\left(\left\|\boldsymbol{\xi}_{h}^{*}-\boldsymbol{\xi}\right\|+\left\|T\left(\boldsymbol{u}_{h}-\boldsymbol{u}\right)\right\|+\left\|\left(T_{h}^{*}-T\right) \boldsymbol{u}_{h}\right\|\right) .
$$

Now, we have using (3.6):

$$
\left\|\left(T_{h}^{*}-T\right) \boldsymbol{u}_{h}\right\| \leq\left\|\boldsymbol{u}_{h}\right\|_{L^{\infty}}\left\|T_{h}^{*}-T\right\| \leq C h|T|_{1, \Omega},
$$

and

$$
\begin{aligned}
\left\|T\left(\boldsymbol{u}_{h}-\boldsymbol{u}\right)\right\| & \leq\|T\|_{L^{\infty}}\left(\left\|\boldsymbol{u}_{h}^{*}-\boldsymbol{u}_{h}\right\|+\left\|\boldsymbol{u}_{h}^{*}-\boldsymbol{u}\right\|\right) \\
& \leq\|T\|_{L^{\infty}}\left(\left\|\boldsymbol{u}_{h}^{*}-\boldsymbol{u}_{h}\right\|+C h|\boldsymbol{u}|_{1, \Omega}\right) .
\end{aligned}
$$

Using these estimates, the fact that $\left\|\boldsymbol{\xi}_{h}^{*}-\boldsymbol{\xi}\right\| \leq C h|\boldsymbol{\xi}|_{1, \Omega}$, and (4.13), we get

$$
\left\|T_{h}^{*}-T_{h}\right\| \leq C\left(h\left(|\boldsymbol{\xi}|_{1, \Omega}+|T|_{1, \Omega}\right)+h\|T\|_{L^{\infty}}|\boldsymbol{u}|_{1, \Omega}+\|T\|_{L^{\infty}}\left\|\boldsymbol{u}_{h}^{*}-\boldsymbol{u}_{h}\right\|\right) .
$$

This implies

$$
\left\|T_{h}^{*}-T_{h}\right\| \leq C R\left(h+\left\|\boldsymbol{u}_{h}^{*}-\boldsymbol{u}_{h}\right\|\right)
$$


and, by (4.12), we get

$$
\left\|\boldsymbol{\xi}_{h}^{*}-\boldsymbol{\xi}_{h}\right\| \leq C R\left(h+\left\|\boldsymbol{u}_{h}^{*}-\boldsymbol{u}_{h}\right\|\right) .
$$

Now, following the discrete inf-sup condition and (4.3), we obtain

$$
\begin{aligned}
& \beta^{*}\left(\left\|\boldsymbol{u}_{h}^{*}-\boldsymbol{u}_{h}\right\|+\left\|\omega_{h}^{*}-\omega_{h}\right\|\right) \leq \sup _{\boldsymbol{\tau} \in \Sigma_{h}} \frac{\left(\operatorname{div}\left(\boldsymbol{\tau}_{N}+\boldsymbol{\tau}_{P}-q I\right), \boldsymbol{u}_{h}^{*}-\boldsymbol{u}_{h}\right)+\left(\operatorname{as}\left(\boldsymbol{\tau}_{N}\right), \omega_{h}^{*}-\omega_{h}\right)}{\|\underset{\sim}{\boldsymbol{\tau}}\|_{\Sigma}} \\
& =\sup _{\underset{\sim}{\boldsymbol{\tau}} \in \Sigma_{h}} \frac{1}{\|\underset{\sim}{\boldsymbol{\tau}}\|_{\Sigma}}\left\{\begin{array}{l}
\left(\frac{1}{2 \varepsilon \alpha_{N}\left(T_{h}\right)} \boldsymbol{\sigma}_{N h}-\frac{1}{2 \varepsilon \alpha_{N}(T)} \boldsymbol{\sigma}_{N}, \boldsymbol{\tau}_{N}\right)+ \\
\left(\frac{1}{2 \alpha_{P}\left(T_{h}\right)} \boldsymbol{\sigma}_{P h}-\frac{1}{2 \alpha_{P}(T)} \boldsymbol{\sigma}_{P}, \boldsymbol{\tau}_{P}\right)+ \\
\left(a s\left(\boldsymbol{\tau}_{N}\right), \omega_{h}^{*}-\omega\right)
\end{array}\right\} .
\end{aligned}
$$

Since,

$$
\frac{1}{2 \varepsilon \alpha_{N}\left(T_{h}\right)} \boldsymbol{\sigma}_{N h}-\frac{1}{2 \varepsilon \alpha_{N}(T)} \boldsymbol{\sigma}_{N}=\left(\frac{1}{2 \varepsilon \alpha_{N}\left(T_{h}\right)}-\frac{1}{2 \varepsilon \alpha_{N}(T)}\right) \boldsymbol{\sigma}_{N}+\frac{1}{2 \varepsilon \alpha_{N}\left(T_{h}\right)}\left(\boldsymbol{\sigma}_{N h}-\boldsymbol{\sigma}_{N}\right)
$$

and

$$
\frac{1}{2 \alpha_{P}\left(T_{h}\right)} \boldsymbol{\sigma}_{P h}-\frac{1}{2 \alpha_{P}(T)} \boldsymbol{\sigma}_{P}=\left(\frac{1}{2 \alpha_{P}\left(T_{h}\right)}-\frac{1}{2 \alpha_{P}(T)}\right) \boldsymbol{\sigma}_{P}+\frac{1}{2 \alpha_{P}\left(T_{h}\right)}\left(\boldsymbol{\sigma}_{P h}-\boldsymbol{\sigma}_{P}\right),
$$

one gets

$$
\begin{aligned}
\left\|\boldsymbol{u}_{h}^{*}-\boldsymbol{u}_{h}\right\|+\left\|\omega_{h}^{*}-\omega_{h}\right\| \leq & C\left\{\left\|\frac{1}{\alpha_{N}\left(T_{h}\right)}-\frac{1}{\alpha_{N}(T)}\right\|\left\|\boldsymbol{\sigma}_{N}\right\|_{L^{\infty}}+\left\|\boldsymbol{\sigma}_{N h}-\boldsymbol{\sigma}_{N}\right\|+\right. \\
& \left.\left\|\frac{1}{\alpha_{P}\left(T_{h}\right)}-\frac{1}{\alpha_{P}(T)}\right\|\left\|\boldsymbol{\sigma}_{P}\right\|_{L^{\infty}}+\left\|\boldsymbol{\sigma}_{P h}-\boldsymbol{\sigma}_{P}\right\|+\left\|\omega_{h}^{*}-\omega\right\|\right\}, \\
\leq & C\left\{\left\|\boldsymbol{\sigma}_{N}\right\|_{L^{\infty}}\left\|T_{h}-T\right\|+\left\|\boldsymbol{\sigma}_{N h}-\boldsymbol{\sigma}_{N}\right\|+\right. \\
& \left.\left\|\boldsymbol{\sigma}_{P}\right\|_{L^{\infty}}\left\|T_{h}-T\right\|+\left\|\boldsymbol{\sigma}_{P h}-\boldsymbol{\sigma}_{P}\right\|+\left\|\omega_{h}^{*}-\omega\right\|\right\}, \\
\leq & C\left\{\left(\left\|\boldsymbol{\sigma}_{N}\right\|_{L^{\infty}}+\left\|\boldsymbol{\sigma}_{P}\right\|_{L^{\infty}}\right)\left(\left\|T_{h}^{*}-T_{h}\right\|+\left\|T_{h}^{*}-T\right\|\right)+\right. \\
& \left\|\boldsymbol{\sigma}_{N h}-\boldsymbol{\sigma}_{N h}^{*}\right\|+\left\|\boldsymbol{\sigma}_{P h}-\boldsymbol{\sigma}_{P h}^{*}\right\|+\left\|\boldsymbol{\sigma}_{N h}^{*}-\boldsymbol{\sigma}_{N}\right\|+ \\
& \left.\left\|\boldsymbol{\sigma}_{P h}^{*}-\boldsymbol{\sigma}_{P}\right\|+\left\|\omega_{h}^{*}-\omega\right\|\right\} .
\end{aligned}
$$

Then, using (4.2) together with

$$
\left\|T_{h}^{*}-T\right\| \leq C h|T|_{1, \Omega} \text { and }\left\|\omega_{h}^{*}-\omega\right\| \leq C h|\omega|_{1, \Omega}
$$


one gets

$$
\left\|\boldsymbol{u}_{h}^{*}-\boldsymbol{u}_{h}\right\|+\left\|\omega_{h}^{*}-\omega\right\| \leq C R\left(h+\left\|T_{h}^{*}-T_{h}\right\|\right)+C\left(\left\|\boldsymbol{\sigma}_{N h}-\boldsymbol{\sigma}_{N h}^{*}\right\|+\left\|\boldsymbol{\sigma}_{P h}-\boldsymbol{\sigma}_{P h}^{*}\right\|\right) .
$$

From (4.14) and (4.16), we have, for $R$ small enough,

$$
\left\|T_{h}^{*}-T_{h}\right\| \leq C R\left\|T_{h}^{*}-T_{h}\right\|+C R\left(h+\left\|\boldsymbol{\sigma}_{N h}-\boldsymbol{\sigma}_{N h}^{*}\right\|+\left\|\boldsymbol{\sigma}_{P h}-\boldsymbol{\sigma}_{P h}^{*}\right\|\right)
$$

and then,

$$
\left\|T_{h}^{*}-T_{h}\right\| \leq C R\left(h+\left\|\boldsymbol{\sigma}_{N h}-\boldsymbol{\sigma}_{N h}^{*}\right\|+\left\|\boldsymbol{\sigma}_{P h}-\boldsymbol{\sigma}_{P h}^{*}\right\|\right) .
$$

Then, using (4.9), we obtain

$$
\left\|T_{h}^{*}-T_{h}\right\| \leq C R h+C R\left\|T_{h}^{*}-T_{h}\right\| .
$$

Thus, for $R$ small enough, we get the following estimate

$$
\left\|T_{h}^{*}-T_{h}\right\| \leq C h
$$

Now, from (4.9) and (4.17), we have

$$
\left\|\boldsymbol{\sigma}_{N h}-\boldsymbol{\sigma}_{N h}^{*}\right\|+\left\|\boldsymbol{\sigma}_{P h}-\boldsymbol{\sigma}_{P h}^{*}\right\| \leq C h .
$$

By (4.16), (4.17) and (4.18), we obtain

$$
\left\|\boldsymbol{u}_{h}^{*}-\boldsymbol{u}_{h}\right\|+\left\|\omega_{h}^{*}-\omega\right\| \leq C h
$$

and from (4.15), (4.19), we get

$$
\left\|\boldsymbol{\xi}_{h}^{*}-\boldsymbol{\xi}_{h}\right\| \leq C h .
$$

Now, from (4.7), we get $\boldsymbol{\sigma}_{\sim}-\boldsymbol{\sigma}_{\sim}{ }^{*}$ in the discrete kernel and then

$$
\left\|p_{h}^{*}-p_{h}\right\| \leq C\left(\left\|\boldsymbol{\sigma}_{N h}-\boldsymbol{\sigma}_{N h}^{*}\right\|+\left\|\boldsymbol{\sigma}_{P h}-\boldsymbol{\sigma}_{P h}^{*}\right\|\right)
$$

which implies (owing to (4.18))

$$
\left\|p_{h}^{*}-p_{h}\right\| \leq C h
$$

Finally, the error estimates given in the theorem are direct consequences of (4.17)-(4.21), the triangle inequality, together with (4.2) and classical interpolation errors.

\section{Conclusion}

We presented, in this work, a dual mixed formulation of Non-isothermal Oldroyd-Stokes problem. In comparison with the standard finite elements formulations, this approach makes it possible to obtain fine approximations of the dual variables (Newtonian and elastic components of the extra-stress tensor as well as the heat flux). The obtained formulation has local conservation properties. Moreover, after linearization, one can use the hybridization technique (cf., e.g., [12]). This procedure enables us to reduce the algebraic systems sizes. 


\section{Acknowledgment}

The authors would like to thank the anonymous referees for their meticulous work, comments and suggestions.

\section{References}

[1] D.N. Arnold, F. Brezzi, J. Douglas. PEERS: A new mixed finite element for plane elasticity. Japan J. Appl. Math., 1 (1984), 347-367.

[2] J. Baranger, D. Sandri. A formulation of Stokes's problem and the linear elasticity equations suggested by the Oldroyd model for viscoelastic flow. $\mathrm{M}^{2} \mathrm{AN}, 26$ (1992), 331-345.

[3] F. Brezzi, M. Fortin. Mixed and Hybrid Finite Element Methods. Springer-Verlag, Berlin, 1991.

[4] P.G. Ciarlet. The Finite Element Method for Elliptic Problems. North-Holland, 1978.

[5] P. Clément. Approximation by finite element functions using local regularization. RAIRO Anal. Numer., 2 (1975), 77-84.

[6] C. Cox, H. Lee, D. Szurley. Finite element approximation of the non-isothermal StokesOldroyd equations. Int. J. Numer. Anal. Mod., 4 (2007), 425-440.

[7] S. Damak Besbes, C. Guillopé. Non-isothermal flows of viscoelastic incompressible fluids. Nonlinear Analysis, 44 (2001), 919-942.

[8] J. Douglas, Jr., J.E. Roberts. Global estimates for mixed methods for second order elliptic equations. Math. Comp., 44 (1985), 39-52.

[9] L.C. Evans. Partial Differential Equations. Graduate Studies in Mathematics, Vol. 19, American Mathematical Society, Providence, RI, 1999.

[10] M. Farhloul, M. Fortin. A new mixed finite element for the Stokes and elasticity problems. SIAM J. Numer. Anal., 30 (1993), 971-990.

[11] M. Farhloul, M. Fortin. Dual hybrid methods for the elasticity and the Stokes problems: a unified approach. Numer. Math., 76 (1997), 419-440.

[12] M. Farhloul, A.M. Zine. A new mixed finite element method for the Stokes problem. J. Math. Anal. Appl., 276 (2002), 329-342.

[13] P. Grisvard. Problèmes aux limites dans les polygones, mode d'emploi. EDF Bull. Direction Etudes Rech. Sér. C Math. Inform., 1 (1986), 21-59.

[14] J.C. Nedelec. Mixed finite elements in $\mathbb{R}^{3}$. Numer. Math., 35 (1980), 315-341. 
[15] G.W.M. Peters, F.T.O. Baaijens. Modelling of non-isothermal viscoelastic flow. J. NonNewtonian Fluid Mech., 68 (1997), 205-224.

[16] P.A. Raviart, J.M. Thomas. A mixed finite element method for 2nd order elliptic problems, Lecture Notes in Mathematics, Vol. 606, Springer-Verlag, New-York, 1977, pp. 292-315.

[17] J.E. Roberts, J.M. Thomas. Mixed and hybrid finite element methods, Handbook of Numerical Analysis, vol. II, Finite Element Methods (part I), P.G Ciarlet, J.L. Lions (Eds.), North-Holland, 1989. 